\title{
Strand-Specific miR-28-5p and miR-28-3p Have Distinct Effects in Colorectal Cancer Cells
}

\begin{abstract}
MARIA I. ALMEIDA, ${ }^{, \neq}$MILENA S. NICOLOSO, ${ }^{*}$ LIZHI ZENG, ${ }^{\S}$ CRISTINA IVAN," RICCARDO SPIZZO,* ROBERTA GAFÀ, ${ }^{\natural}$

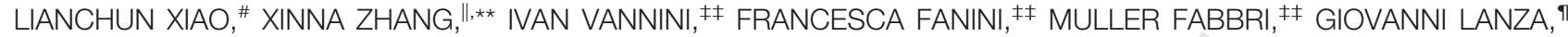
RUI M. REIS, ${ }^{\ddagger, \S \S}$ PATRICK A. ZWEIDLER-MCKAY,,,\|\|, , ๆ and GEORGE A. CALIN ${ }^{*, \|}$

*Department of Experimental Therapeutics, The University of Texas MD Anderson Cancer Center, Houston, Texas; ${ }^{\ddagger}$ Life and Health Sciences Research Institute, School of Health Sciences, University of Minho, Braga, Portugal; Departments of ${ }^{\text {SPediatrics and }{ }^{\prime \prime} T h e}$ Center for RNA Interference and Non-Coding RNAs, The University of Texas MD Anderson Cancer Center, Houston, Texas; "Department of Experimental and Diagnostic Medicine and Interdepartmental Center for Cancer Research, University of Ferrara, Ferrara, Italy; "Division of Quantitative Science and **Department of Gynecologic Oncology, The University of Texas MD Anderson Cancer Center, Houston, Texas; ${ }^{* \neq}$ Istituto Scientifico Romagnolo per lo Studio e la Cura dei Tumori, Meldola, Italy; \$\$ Molecular Oncology Research Center, Barretos Cancer Hospital, Barretos, Sao Paulo, Brazil; "IIThe Metastasis Research Center, The University of Texas MD Anderson Cancer Center, Houston, Texas; $191 /$ The University of Texas Graduate School of Biomedical Sciences, Houston, Texas
\end{abstract}

BACKGROUND \& AIMS: MicroRNAs (miRs) can promote or inhibit tumor growth and are therefore being developed as targets for cancer therapies. They are diverse not only in the messenger RNAs (mRNA) they target, but in their production; the same hairpin RNA structure can generate mature products from each strand, termed $5 p$ and $3 p$, that can bind different mRNAs. We analyzed the expression, functions, and mechanisms of miR-28-5p and miR-28-3p in colorectal cancer (CRC) cells. METHODS: We measured levels of miR-28-5p and miR-28-3p expression in 108 CRC and 49 normal colorectal samples (47 paired) by reverse transcription, quantitative real-time polymerase chain reaction. The roles of miR-28 in CRC development were studied using cultured HCT116, RKO, and SW480 cells and tumor xenograft analyses in immunodeficient mice; their mRNA targets were also investigated. RESULTS: miR-28-5p and miR-28-3p were downregulated in CRC samples compared with normal colon samples. Overexpression of miRs in CRC cells had different effects and the miRs interacted with different mRNAs: miR-28-5p altered expression of CCND1 and HOXB3, whereas miR-28-3p bound NM23-H1. Overexpression of miR-28-5p reduced CRC cell proliferation, migration, and invasion in vitro, whereas miR-28-3p increased CRC cell migration and invasion in vitro. CRC cells overexpressing miR-28 developed tumors more slowly in mice compared with control cells, but miR-28 promoted tumor metastasis in mice. CONCLUSION: miR-28-5p and miR-28-3p are transcribed from the same RNA hairpin and are down-regulated in CRC cells. Overexpression of each has different effects on CRC cell proliferation and migration. Such information has a direct application for the design of miR gene therapy trials.

Keywords: Transcript Regulation; Gene; RNA Processing.

C olorectal (CRC) cancer is the third most commonly diagnosed cancer in men and the second in women. ${ }^{1}$ In the United States, it is the third leading cause of death by cancer, with 51,371 estimated deaths and 142,570 estimated newly diagnosed cases in $2010 .^{2}$ Therefore, new therapeutic approaches and prognostic markers are needed. In 2002, new players in cancer biology were identified: microRNAs (miRNAs). ${ }^{3}$ These are a large family of AQ: 7 small noncoding RNAs with approximately 20 -nt length that regulate gene expression post-transcriptionally by inhibition of translation or messenger RNA (mRNA) degradation. ${ }^{4}$ miRNAs targeting occurs by binding to $3^{\prime}$ untranslated regions, coding sequences, or $5^{\prime}$-untranslated regions of target mRNA that can be involved in diverse biological processes, such as proliferation, apoptosis, inflammation, differentiation, and metastasis. ${ }^{4}$ miRNAs can function as either oncogenes or tumor suppressor genes, depending on the type of tumor or the cellular context. ${ }^{5}$ In CRC, miRNAs have been involved in tumor susceptibility (as polymorphisms in miRNA-binding sites have been associated with CRC risk) and in diagnosis (as miRNAs can be detected in feces or blood and used as biomarkers). ${ }^{6}$ In addition, miRNA expression is dysregulated in CRC, as well as in other cancer types, and miRNAs have emerged as potential new therapeutic targets. ${ }^{6,7}$ Therefore, understanding the role of miRNAs in CRC is crucial for the development of new therapies.

In the miRNA biogenesis pathway, long primary transcripts transcribed from the genome are processed by the cellular RNase enzyme III Drosha into a structure of 60 to $110 \mathrm{nt}$ called precursor miRNA (pre-miRNA), which is then exported to the cytoplasm by an Exportin 5-dependent mechanism. ${ }^{4}$ The pre-miRNA is cleaved by the RNase III enzyme Dicer-1 producing a short, imperfect, doublestranded miRNA duplex, which is unwound by a helicase, creating a mature miRNA. ${ }^{4}$ In some cases, 2 mature miRNAs can be excised from the same stem-loop pre-miRNA. ${ }^{8}$ These $5 \mathrm{p}$ and $3 \mathrm{p}$ miRNAs, although generated from a single primary transcript, have different sequences and therefore tar-

\footnotetext{
Abbreviations used in this paper: CRC, colorectal cancer; miRNA, microRNA; MSS, microsatellite stable; mRNA, messenger RNA; MSI, microsatellite unstable; PCR, polymerase chain reaction; SCR, scrambled control; PARP1, poly(adenosine diphosphate-ribose) polymerase 1; pre-miRNA, precursor miRNA.
}

$$
\begin{gathered}
\text { (c) } 2012 \text { by the AGA Institute } \\
0016-5085 / \$ 36.00
\end{gathered}
$$

doi:10.1053/j.gastro.2011.12.047

\section{1}

3

\section{4}

6

7 
get different mRNAs. In humans, 2 different mature miRNA sequences are excised from opposite arms of the stem-loop pre-miR-28 and generate 2 different miRNAs-hsa-miR28-5p and hsa-miR-28-3p. Despite nearly a decade of studies on miRNA roles in cancer, ${ }^{3}$ the comparative roles of strandspecific mature miRNAs that originated from the same stem-loop precursor ( $5 p$ and $3 p$ ) have not yet been fully studied.

To our knowledge, the roles miR-28-5p and miR-28-3p play in CRC has never been described. Therefore, the purpose of our study was to analyze miR-28-5p and miR28-3p expression and to use in vitro and in vivo approaches to understand, for the first time, the functions and mechanisms of these 2 miRNAs in CRC.

\section{Materials and Methods Colorectal Samples}

Eighty-five CRC samples and 26 normal colorectal tissue samples (of which 24 were paired) were collected between 2003 and 2008 at the University Hospital of Ferrara in Ferrara, Italy (first sample set). Forty-two tumors were classified as microsatellite stable (MSS), and 43 tumors were classified as microsatellite unstable (MSI) (Supplementary Methods). For a confirmation set of samples, we obtained 23 paired samples of tumor and adjacent colorectal tissue that were collected between 2002 and 2005 at the Istituto per lo Studio e la Cura dei Tumori della Romagna in Meldola, Italy (second sample set). Tumors were classified according to the World Health Organization pathologic classification system. All patients provided informed consent, and collection of the samples was approved by the institutional review board at each institution. Patients did not receive any therapy before surgery. Upon resection, fresh surgical specimens were immediately snap-frozen in liquid nitrogen and stored at $-80^{\circ} \mathrm{C}$. Total RNA from tissue samples was isolated using Trizol reagent (Invitrogen, Carlsbad, CA), according to manufacturer's instructions (Supplementary Methods).

\section{Reverse Transcription Quantitative Real-Time Polymerase Chain Reaction}

RNA purity was assessed by measuring absorbance at 260, 280, and $230 \mathrm{~nm}$. Mean 260/280 ratio was $1.97 \pm 0.05$, with a range between 1.86 and 2.05 , and mean 260/230 ratio was $2.17 \pm$ 0.11 , with a range between 2.00 and 2.31. In addition, as recommended by the Minimum Information for Publication of Quantitative Real-Time PCR Experiments guidelines, ${ }^{9,10}$ we analyzed RNA integrity by gel electrophoresis and clearly defined $28 \mathrm{~S}$ and $18 \mathrm{~S}$ ribosomal RNA bands were visualized. Samples with low quality that did not meet these criteria were excluded. We quantified miR-28-5p and miR-28-3p expression with real-time quantitative polymerase chain reaction TaqMan miRNA assays (Applied Biosystems, Foster City, CA), namely assay 000411 for miR-28-5p, assay 002446 for miR-28-3p, and assay 001973 for U6 snRNA (Supplementary Methods). The efficiency of the Taqman assays used in this study was determined (Supplementary Figure 1 and Supplementary Table 1). Relative expression levels were calculated using the $\Delta \Delta \mathrm{C}_{\mathrm{t}}{ }^{11}$ and the Pfaffl method. ${ }^{12}$

\section{In Vitro Cell Proliferation Assays}

HCT116 and RKO cells transfected with scrambled control (SCR), miR-28-5p, or miR-28-3p were seeded onto a 12-well plate at $1 \times 10^{5}$ cells/well in triplicate. Cells were harvested and counted at $0,24,48,72$, and 96 hours after transfection using the Vi-CELL cell viability analyzer (Beckman Coulter, Brea, CA). In order to further confirm our results, a 3-(4,5-dmethylthiazol2-yl)-2,5-diphenyltetrazolium bromide assay was performed (Supplementary Methods). The experiment was repeated twice independently.

\section{In Vitro Cell Migration and Invasion Assays}

To determine the effect of miR-28-5p and miR-28-3p on cell migration, we used $6.5-\mathrm{mm}$ diameter Transwell chambers with $8-\mu \mathrm{m}$ pore size polycarbonate membranes (Corning Incorporated, Lowell, MA). To determine the effect of these miRNAs on cell invasion, we used BioCoat growth-factor reduced Matrigel invasion chambers (BD Biosciences, Bedford, MA). Cells transfected with SCR, miR-28-5p, or miR-28-3p were resuspended in serum-free medium and plated on the top of the Transwell chambers. Fetal bovine serum was used as a chemoattractant on both assays. Each assay was performed in triplicate and in 2 independent experiments. Additional details are described in Supplementary Methods.

\section{In Vivo Studies of Tumorigenesis and Metastatic Potential}

For the in vivo tumorigenesis assay, $1.5 \times 10^{6}$ HCT116pBABE-miR28 or HCT116-pBABE-empty cells were subcutaneously injected into the flanks of NOD-SCID-IL2R-deficient mice ( $\mathrm{n}=9$; stock \#005557; The Jackson Laboratory, Bar Harbor, ME). Tumor size was measured every 2 days. Animals were sacrificed 21 days after injection, and final tumor volume was determined. Tumor size was determined by digital caliper measurements (length and width in $\mathrm{mm}$ ), and tumor volume $\left(\mathrm{mm}^{3}\right)$ was estimated using the following formula: tumor volume $=1 / 2$ (length $\times$ width $^{2}$ ).

For the in vivo tumor-metastasis assay, $4 \times 10^{6}$ HCT116pBABE-miR28 and HCT116-pBABE-empty cells were injected into the tail vein of NOD-SCID-IL2R-deficient mice $(\mathrm{n}=11$ / group). Thirty-five days after injection the mice were sacrificed. All of the organs were examined at necropsy. Tumors were sectioned, stained with $\mathrm{H} \& \mathrm{E}$, and anti-green fluorescent protein antibody (Ab13970; Abcam, Cambridge, MA), and examined histologically.

All animal care and handling was approved by The University of Texas MD Anderson Institutional Animal Care and Use Committee.

\section{Statistical Analysis}

Shapiro-Wilk test was used to verify the clinical samples' distribution. Differences were analyzed using the nonparametric test Mann-Whitney-Wilcoxon. To compare the paired groups, paired $t$ test was used. For in vitro and in vivo studies, the differences between groups were analyzed using Student $t$ test (2-tailed), assuming unequal variance. Discrete variables were compared with Fisher exact test. Graphics represent the mean \pm standard deviation, unless otherwise stated. Statistical analysis was performed in $\mathrm{R}$ (version 2.11.0). Statistical significance was considered if $P<.05$.

Additional methods, including cell culture, STR DNA fingerprinting, and miRNA mimics transfection, apoptosis quantification, caspase activity, cell cycle analysis by flow cytometry, establishment of miR-28-expressing cell line, miRNA target prediction, Western blot, and luciferase reporter assays, are available in Supplementary Methods. 
Figure 1. Expression of miR28-5p and miR-28-3p in colon tissue samples. (A) Quantitative realtime PCR analysis shows that miR-28-5p and miR-28-3p are down-regulated in colon cancer samples compared with normal colorectal tissue samples. (B) Both MSS and MSI tumors express significantly less miR-28-5p and miR-28-3p levels when compared with normal colon tissue. No differences were found when comparing miR-28-5p and miR28-3p levels of MSS and MSI tumors. (C) miRNAs down-regulation in CRC tumors paired with normal tissue from the second set of patients. All values of miRNA expression levels were normalized to small nuclear RNA U6. Mean \pm standard error of the mean are represented in the images ${ }^{* \star *} P<$ .005, Mann-Whitney-Wilcoxon test, and paired $t$ test for paired normal vs tumor groups).
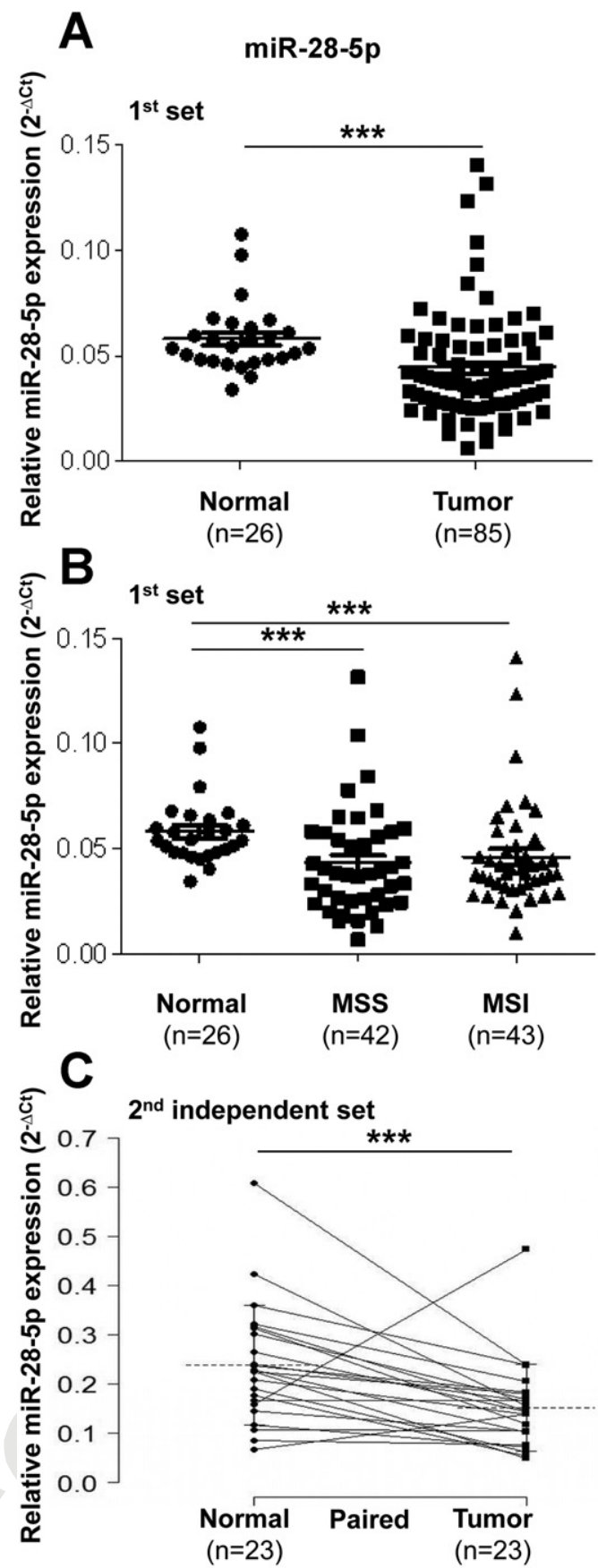

miR-28-3p
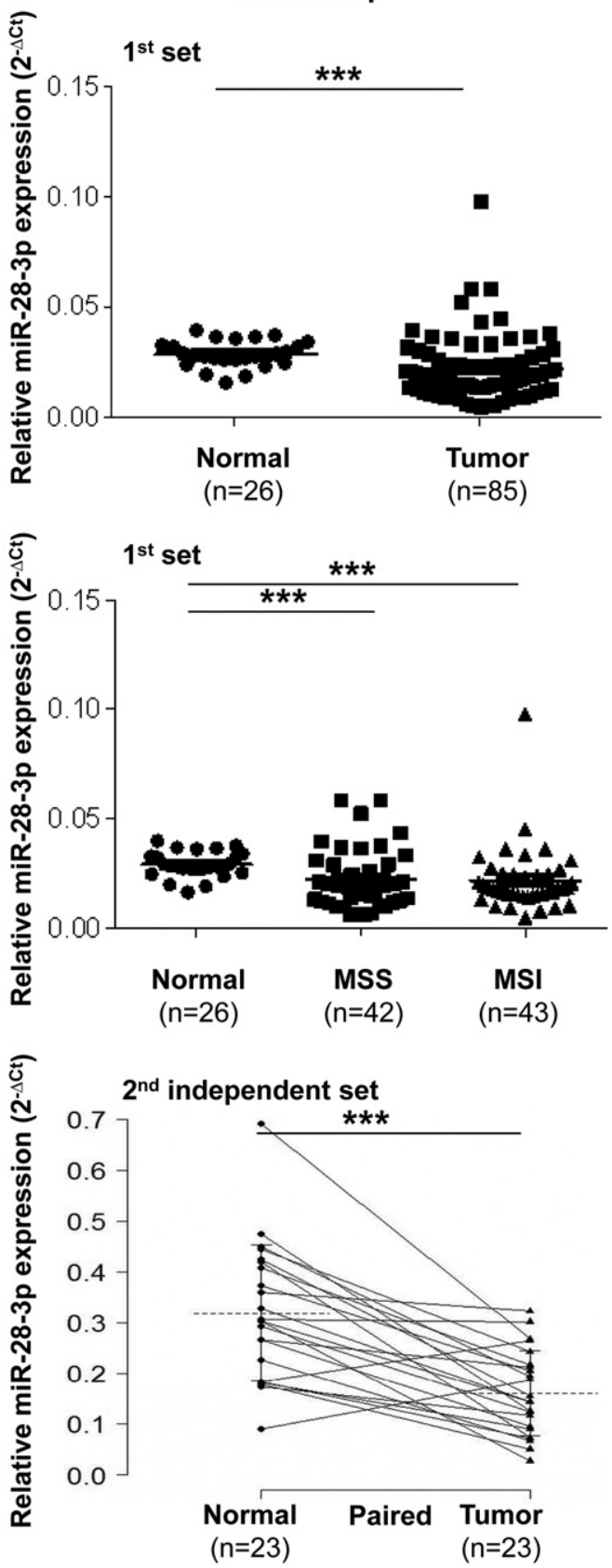
.41) (Supplementary Figure 2). Both miRNA-28-5p and miR-28-3p were significantly down-regulated in CRC samand in agreement with these data, we found significant down-regulation of miR-28-5p and miR-28-3p in CRC 

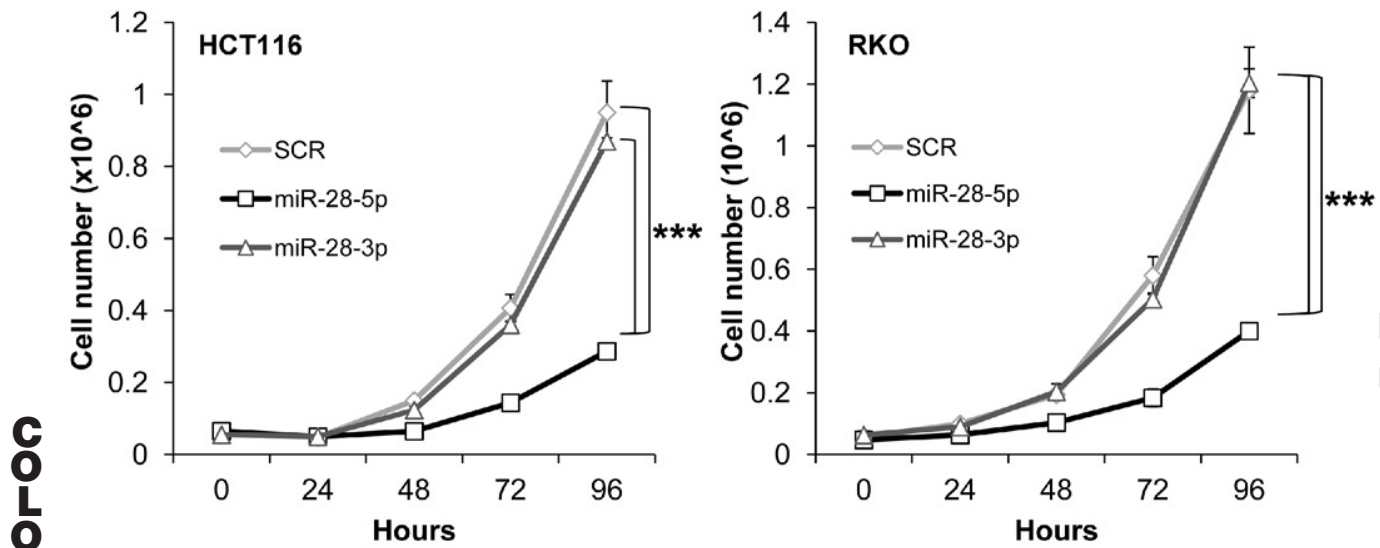

\section{C}
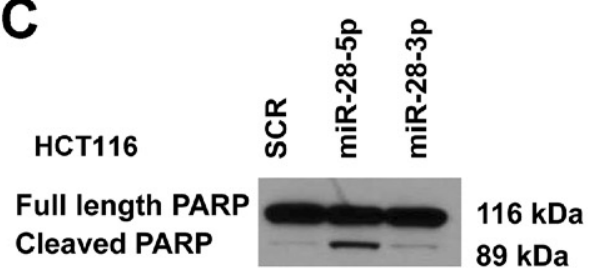

RKO

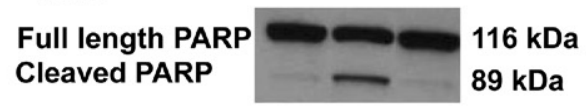

Full length PAR
Cleaved PARP $89 \mathrm{kDa}$

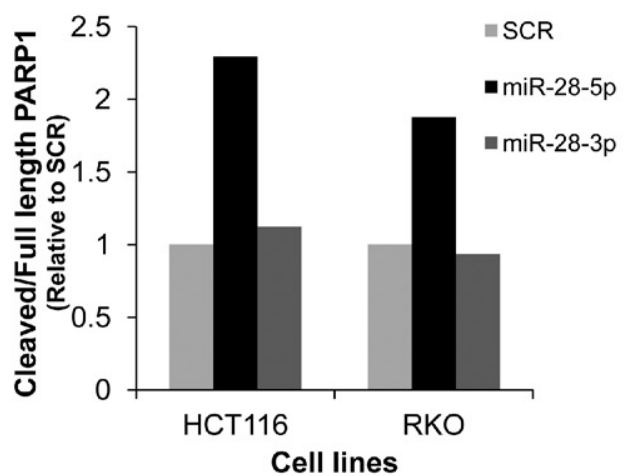

D

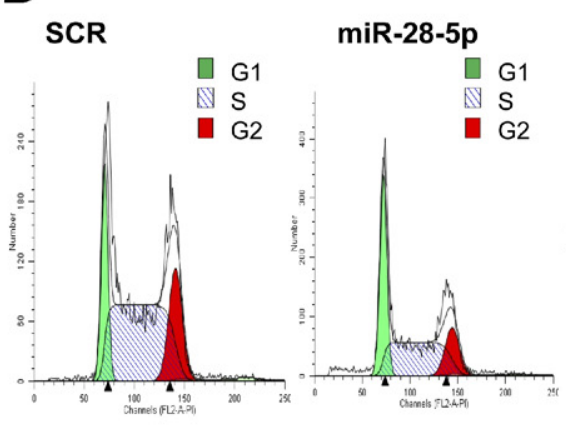

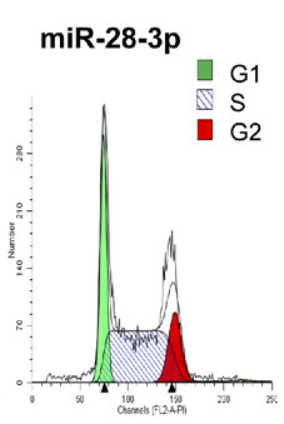

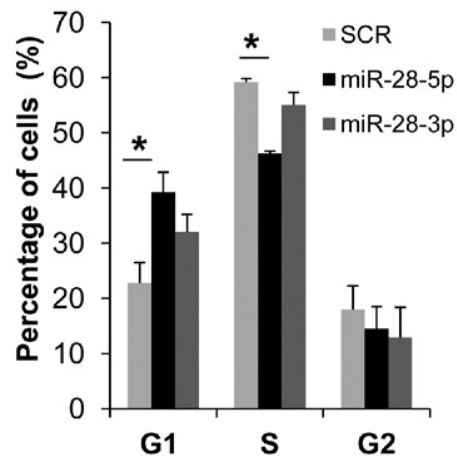

Figure 2. Biological effects of miR-28-5p in proliferation, apoptosis, and cell cycle in vitro. $(A, B)$ Representative experiment of the proliferation effect of miR-28-5p and miR-28-3p in HCT116 and $\mathrm{RKO}$ colon cell lines. Cell numbers were counted every 24 hours for 4 days post-transfection with SCR, miR-28-5p, or miR-28-3p. miR28-5p, but not miR-28-3p, inhibited growth in both HCT116 and RKO cell lines. Values represent the mean of 3 replicates \pm standard deviation $\left({ }^{\star \star \star} P<.005\right.$, Student $t$ test). Two independent experiments were performed. (C) Immunoblotting with anti- PARP1 48 hours after transfection of HCT116 and RKO cell lines with SCR, miR-28-5p, or miR-28-3p. Graphic represents the ratio between cleavage and total PARP1 form. miR-28-5p, but not miR-28$3 p$, increased PARP1 cleavage form. (D) Fluorescent-activated cell sorting analysis 48 hours post-transfection with SCR, miR28-5p, or miR-28-3p. Representative experiment was performed in duplicate; mean \pm standard deviation ( ${ }^{*} P<.05$, Student $t$ test). Two independent experiments were performed. samples (miR-28-5p, $P<.005$; miR-28-3p, $P<.005$ ) (Supplementary Figure 3). In order to confirm these results, we used a second independent set of CRC samples. In 23 paired samples of tumors and adjacent normal tissue, we also found that both miRNAs were downregulated (miR-28-5p, $P<.001$; miR-28-3p, $P<.001$ ) (Figure 1C). Values of expression are presented in Supplementary Tables 2 and 3.

\section{miR-28-5p, but Not miR-28-3p, Significantly Suppresses Proliferation and Induces Apoptosis and G1 Arrest in CRC Cells}

To elucidate the roles of miR-28-5p and miR-28-3p in CRC tumorigenesis, HCT116 and RKO CRC cell lines (endogenous miR-28 expression levels of colon cell lines are shown in Supplementary Figure 4) were transfected with SCR, pre-miR-28-5p, or pre-miR-28-3p. Expression of miRNAs was confirmed by quantitative real-time PCR (Supplementary Figure 5). In both cell lines, we found that cells overexpressing miR-28-5p grew significantly less $(P<.005)$ than did cells transfected with control or miR-28-3p (Figure $2 A$ and $B$ ). This result was also con- F2 firmed in the HCT116 and RKO cell lines using the 3-(4,5-dmethylthiazol-2-yl)-2,5-diphenyltetrazolium bromide assay (Supplementary Figure $6 A$ and $B$ ). In contrast, in both cell lines overexpressing miR-28-3p, there were no statistically significant differences at any time (HCT116, $P=.25$; RKO, $P=.81)$ compared with cells transfected with control (Figure $2 A$ and $B$ ). Therefore, the in vitro results suggest that miR-28-5p, but not miR-28-3p, has a biological effect on proliferation.

We then explored the possibility that the effect of miR-28-5p on proliferation could be due to an increase in 
apoptosis or to defects in the cell cycle. To test whether miR-28-5p had an effect on apoptosis, we measured poly(adenosine diphosphate-ribose) polymerase 1 (PARP1) protein, which is specifically cleaved by caspases and promotes apoptosis. PARP1 cleavage forms are one of the most reliable apoptotic markers. ${ }^{13,14}$ Cells transfected with pre-miR-28-5p expressed 2.2 and 1.8 times more cleaved-PARP1 form (relative to total-PARP1 form) than did cells transfected with control in the HCT116 and RKO cell lines, respectively (Figure 2C). In agreement with the results of the proliferation assays, cells transfected with miR-28-3p presented a PARP1 cleaved to total form ratio similar to the control (Figure $2 C$ ). In addition, our results were confirmed by caspases $3 / 7,8$, and 9 activities, which were all higher in miR-28-5p-transfected cells than in the SCR-transfected cells (Supplementary Figure 6C). In order to analyze possible differences in the cell cycle, the HCT116 cell line was transfected with either SCR, miR-28-5p, or miR-28-3p and analyzed by fluorescentactivated cell sorting. Compared with the control, cells transfected with miR-28-5p had a significantly higher percentage of cells in G1 phase and a significantly lower percentage of cells in $S$ phase, suggesting that miR-28-5p causes $\mathrm{G} 1$ arrest $(P<.05)$ (Figure $2 D)$. Despite being concomitantly transcribed and being part of the same RNA stem-loop hairpin, these data suggest that miR28-5p has a tumor-suppressive role in CRC and that miR-28-3p does not have the same biologic role.

\section{miR-28 Disrupts Tumor Growth In Vivo}

Because our in vitro studies indicated that miR28-5p acts as a tumor suppressor in CRC, we analyzed the overall effect of miR-28 in vivo. For that purpose, we generated stable clones overexpressing miR-28, and expression of miR-28-5p and miR-28-3p was verified by quantitative real-time PCR (Supplementary Figure 7). HCT116 colon cancer cells stably transfected with pBABE-empty or pBABE-miR-28 were subcutaneously injected into the left and right flanks of each mouse, respectively $(\mathrm{n}=9)$. Both cell lines were injected into the same mice to decrease inter-mouse variability. Tumors derived from the HCT116 stably expressing pBABE-miR-28 cells grew much slower than did tumors derived from the F3 HCT116 stably expressing pBABE-empty cells (Figure 3A). Accordingly, final tumor volume in pBABE-miR-28 tumors was significantly reduced $(P<.01)$ compared with pBABE-empty tumors (Figure $3 B$ and $C$ ). miR-28 expression levels were confirmed in these tumors. In pBABEmiR-28 tumors, miR-28-5p and miR-28-3p were increased $(P<.01)$ when compared with pBABE-empty tumors (Figure $3 D$ ). In conclusion, this xenograft experiment revealed that expression of miR-28 disrupts tumor growth in vivo.

\section{Opposite Effects of miR-28-5p and miR-28-3p in Cell Migration and Invasion}

To better understand the biological importance of miR-28-5p and miR-28-3p in CRC, we explored whether these miRNAs could be involved in colon cancer metastasis. To evaluate the migratory capacity of HCT116 cells expressing either miRNA, we used Transwell cell migration assays. Overexpression of miR-28-5p led to a significant reduction in cell migration $(P<.01)$, whereas overexpression of miR-28-3p led to a significant increase $(P<$ .05 ) in cell migration compared with the control (Figure 4). The same result was obtained when using SW480 F4 transfected cells (miR-28-5p, $P<0.05$; miR-28-3p; $P<$ .01) (Supplementary Figure 8). To determine whether both miR-28-5p and miR-28-3p also played a role in invasion, we used Transwell chambers coated with Matrigel. HCT116 cells expressing miR-28-5p had a reduction in invasiveness $(P<.05)$, whereas cells expressing miR$28-3 p$ had an increase in invasiveness $(P<.01)$ compared with the control (Figure 4). Although no statistically significant differences were obtained for SW480 cell line, the same trend was observed-miR-28-5p overexpressing cells are less invasive and miR-28-3p are more invasive than control (miR-28-5p, $P=.25$; miR-28-3p, $P=.12$ ) (Supplementary Figure 8). The effect of miR-28-3p, which showed a growth rate similar to the control, on migration and invasion appears to be independent of cell growth. Therefore, although both miRNAs are down-regulated in $\mathrm{CRC}$, they play different roles in the migration phenotype.

\section{mir-28 Increases Metastasis In Vivo}

As miR-28-5p and miR-28-3p exert opposite effects on migration and invasion in vitro but are transcribed concomitantly in cells, we investigated the effect of global miR-28 expression on metastasis in vivo. For this purpose, we intravenously injected mice with pBABE-empty or pBABE-miR-28 cells. After 35 days, the mice were sacrificed. At necropsy, tumors were found in the liver, kidney, lung, and spinal cord. We found increased number of mice with metastases in all tumor sites in the pBABEmiR-28 group compared with the pBABE-empty group (Figure $5 A$ ). In particular, metastases in the liver and lung were found at a statistically significant higher frequency in the pBABE-miR-28 group than were in the pBABEempty group $(P<.05)$. Examples of tumor metastases from the 3 most frequent locations-liver, kidney, and lung-are presented with H\&E staining and anti-green fluorescent protein labeling (Figure $5 B$ ). In addition, the number of tumors in liver and kidney was higher in the pBABE-miR-28 group than in pBABE-empty (Figure 5C). In particular, in the pBABE-miR-28 group, 6 mice presented liver tumors with a mean of $1.5 \pm 0.8$ tumors per mice, and in the pBABE-empty group, there was only 1 mouse that developed only 1 liver tumor. Regarding the kidney, in the pBABE-miR-28 group, 10 mice presented kidney tumors, with a mean of $14.6 \pm 4.2$ tumors per mice (considering both kidneys), and in the pBABE-empty group, 6 mice developed kidney tumors, with an average of $6 \pm 4.2$ tumors per mice $(P<.005)$. An example of the tumors can be visualized in Figure $5 D$. Although miR28-5p and miR-28-3p had contrasting effects on migration and invasion in vitro, and although in vivo subcuta- 
A

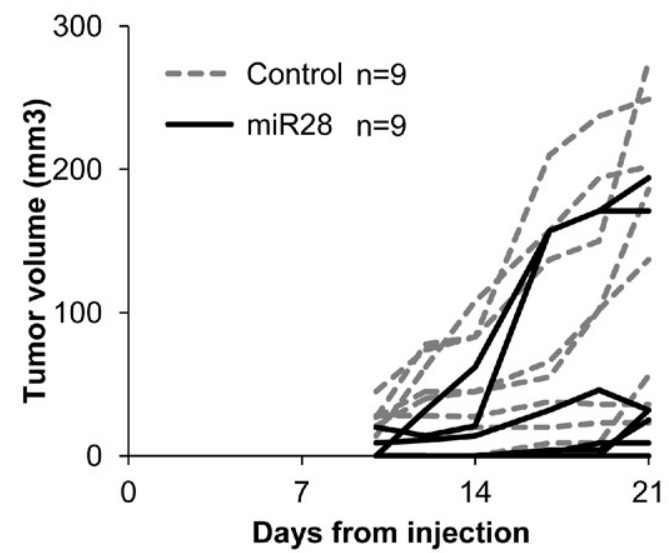

C

\section{Left Flank Empty}

308

309

310
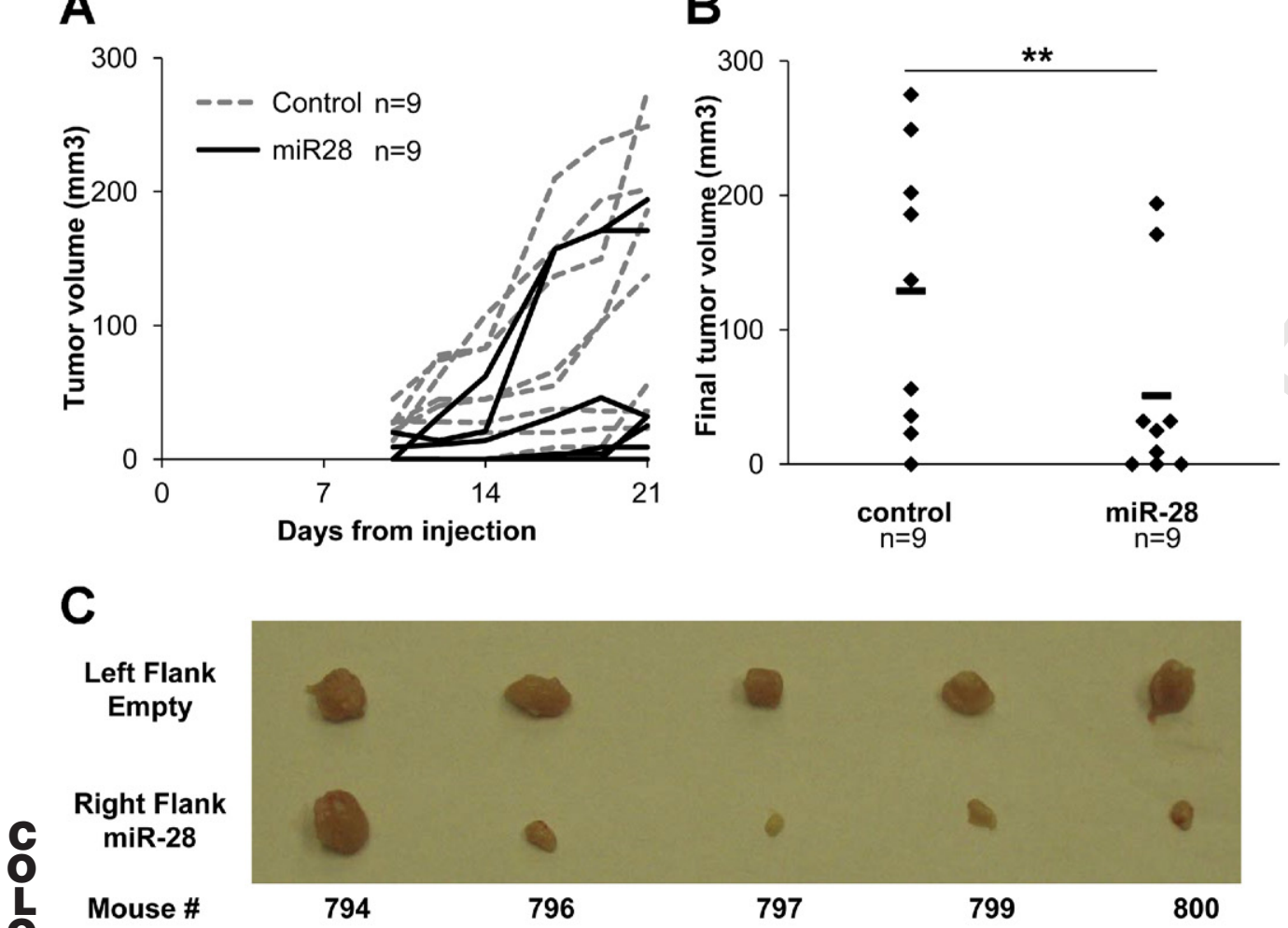

Figure 3. miR-28 decreases tumor volume in mice xenografts. (A, B) HCT116-pBABE-empty (control) and HCT116-pBABEmiR-28 (stably expressing miR28) were subcutaneously injected in the left and right flanks of 9 mice, and tumor volume was measured during the $(A)$ course of the experiment and $(B)$ at the end of the experiment (21 days post inoculation). Tumor volumes in the HCT116-pBABE-miR-28 group were lower than those in the HCT116-pBABE-empty group ${ }^{\star \star} P<.01$, Student $t$ test). (C) Photographs show tumors excised from 5 mice in each group. (D) Quantitative real-time PCR analysis shows miR-28-5p and miR-28-3p expression in the tumors extracted from the mice (mean \pm standard deviation) $\left({ }^{\star \star} P\right.$ $<.01$, Student $t$ test). miR-28-3p in PITA, TargetScan, and miRanda programs simultaneously, we found 5784 mRNAs. Of these mRNAs, 2629 were predicted to be a target of miR-28-5p but not miR-28-3p; 1305 were predicted to be a target of miR-

We first used immunoblotting to detect changes at the protein level for several predicted targets of interest in cells transfected with SCR, miR-28-5p, or miR-28-3p. We 

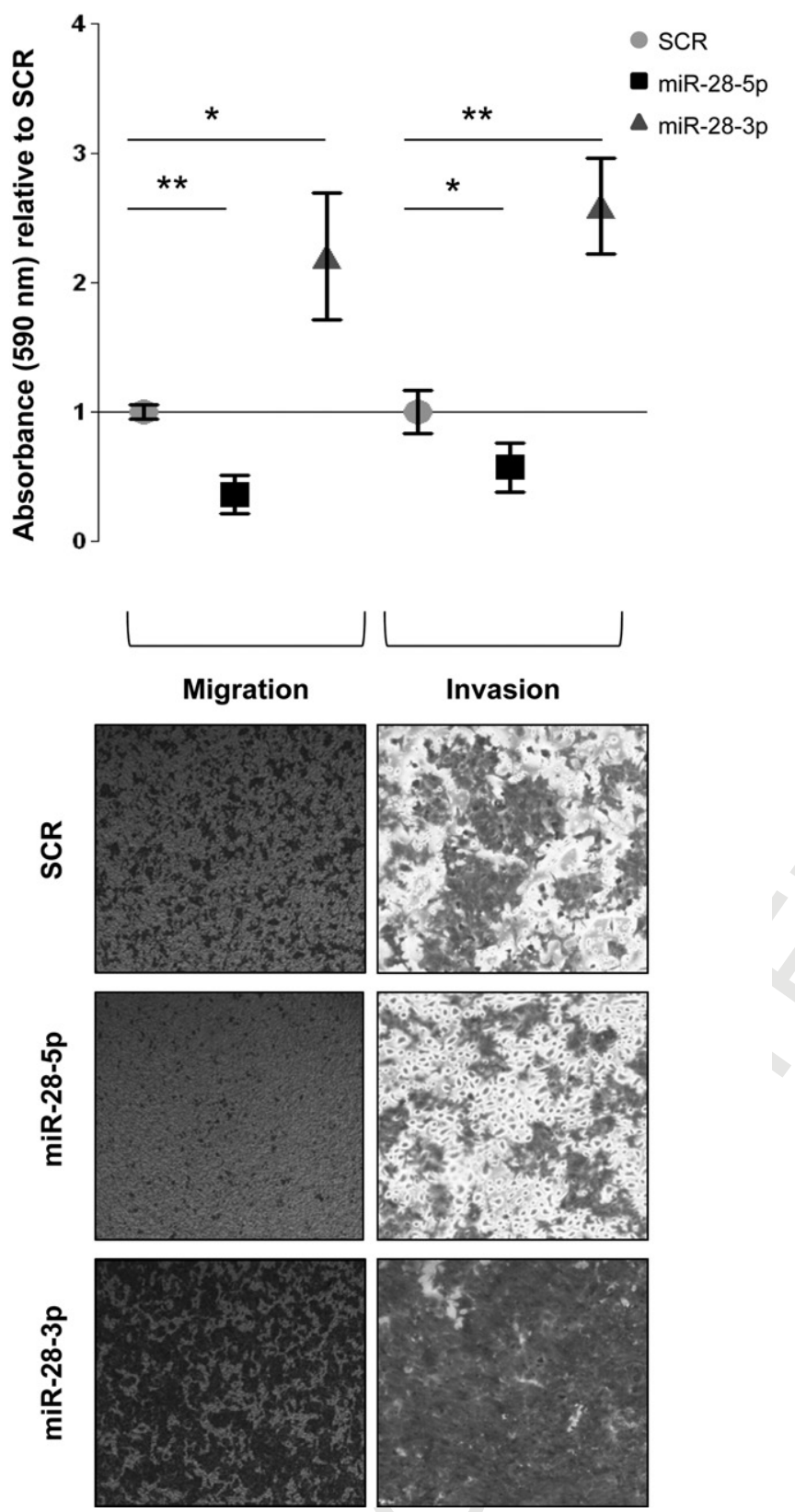

Figure 4. Effect of mir-28-5p and miR-28-3p on migration and invasion in vitro. Absorbance was measured for cells on the bottom of noncoated and Matrigel-coated Transwell chambers at 24 hours (for migration) and 48 hours (for invasion) after HCT116 cells expressing miR-28-5p or miR-28-3p were plated. Results are shown relative to SCR. A representative experiment is shown. Mean of triplicates \pm standard deviation is shown ( ${ }^{*} P<.05 ;{ }^{* *} P<.01$, Student $t$ test). Microscopy images $(\times 50)$ show the migratory and invasive cells on Transwell assays.

found a $51 \%$ reduction in the level of cyclin D1 (encoded by the CCND1 gene) in cells in which miR-28-5p was restored. On the contrary, no differences in cyclin D1 levels were detected in miR-28-3p-expressing cells comF6 pared with SCR-transfected cells (Figure 6A). We also found that $H O X B 3$ was a target of miR-28-5p because this miRNA reduced HoxB3 protein expression by $35 \%$ (Figure $6 B$ ). Regarding miR-28-3p, we found that the protein $\mathrm{Nm} 23-\mathrm{H} 1$ was down-regulated by $52 \%$ in cells expressing miR-28-3p (Figure 6C).
To determine whether the effect on these targets was caused by direct binding of the miRNAs or by an indirect effect, we cloned the predicted mRNA binding sites (Figure $6 D$ and $E$; Supplementary Figure 9) downstream of the modified coding region of firefly luciferase in pGL3 reporter vector. We found that miR-28-5p significantly reduced luciferase activity in the HOXB3 reporter construct by 38\% $(P<.01)$ (Figure $6 D)$. Also, miR-28-3p reduced luciferase activity in the NM23-H1 reporter construct by $34 \%(P<.01)$ (Figure $6 E)$, and no significant differences were found when cells were cotransfected with miR-28-5p and the NM23-H1 construct (Supplementary Figure 10). To confirm this specific interaction, we mutated the miRNA-binding sites, and the luciferase activity for the PGL3-HOXB3 and PGL3-NM23-H1 constructs was restored to the same levels as the control. Regarding CCND1, although we found a significant decrease in luciferase activity in miR-28-5p-transfected cells, the binding site mutation did not fully restore the luciferase activity to the control level (Supplementary Figure 9). In summary, we found that miR-28-5p targeted cyclin D1 and HoxB3 and that miR-28-3p targeted Nm23-H1; this could explain, at least in part, the biological effects observed.

\section{Discussion}

In the present study, we analyzed 2 independent sets of human CRC samples, for a total of 108 (47 paired with normal tissue), and found significant down-regulation of both mature miR-28 forms. Our study is the first to show down-regulation of miR-28 in cancer. In the literature, only 1 study extensively analyzed miR-28 function in cancer, namely in myeloproliferative neoplasms. Girardot et al identified miR-28 overexpression in platelets of BCR-ABL-negative myeloproliferative neoplasm patients and found MLP to be the main target, which is important for megakaryocyte differentiation. ${ }^{15}$ In normal colon tissue, in situ hybridization shows that miR-28-5p and miR-28-3p are predominantly expressed in epithelial cells (Supplementary Figure 11). In addition, a couple of profiling studies showed miR-28 up-regulation in renal cell carcinoma ${ }^{16}$ and during glioma progression. ${ }^{17}$ It is well established that miRNAs can function as either tumor suppressors or oncogenes, depending on the tumor tissue and the cell type. ${ }^{5}$ Therefore, when studying miRNAs, it is essential to take into consideration the cellular context. ${ }^{5,18}$ One of the best examples is miR$125 \mathrm{a} / \mathrm{b}$, which has been shown to be down-regulated in glioblastoma, breast, prostate, ovarian, and non-small cell lung cancer, but up-regulated in myelodysplastic syndrome and acute myeloid leukemia patients with $\mathrm{t}(2 ; 11)(\mathrm{p} 21 ; \mathrm{q} 23)$ and in urothelial carcinoma. ${ }^{18,19}$ Noteworthy, miRNA variation levels between normal tissue and tumors of $<50 \%$ are reported frequently, and Volinia et al, who represent the largest miRNA profiling study reported so far, shows as highly significant consistent variations of $<20 \%{ }^{20}$

As down-regulation miR-28-5p and miR-28-3p had never been described before, we analyzed their roles in 
406
A
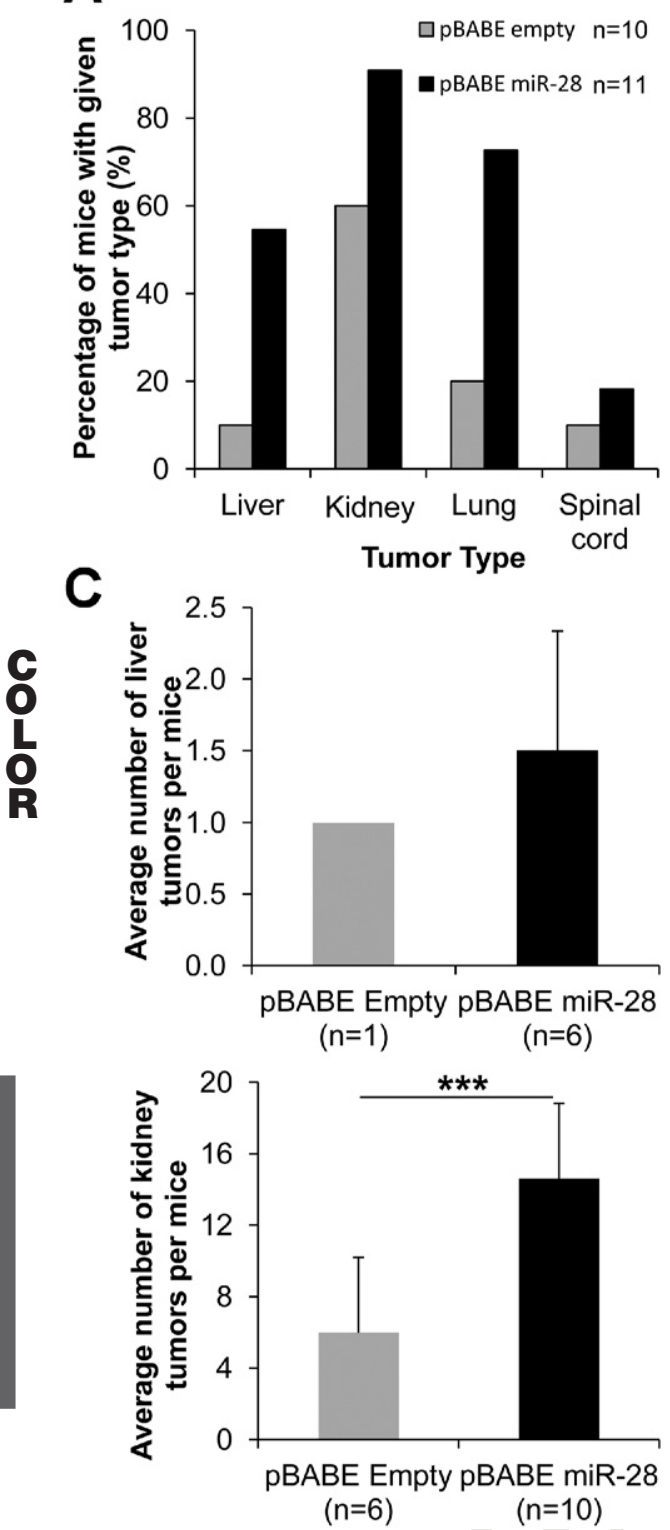

B
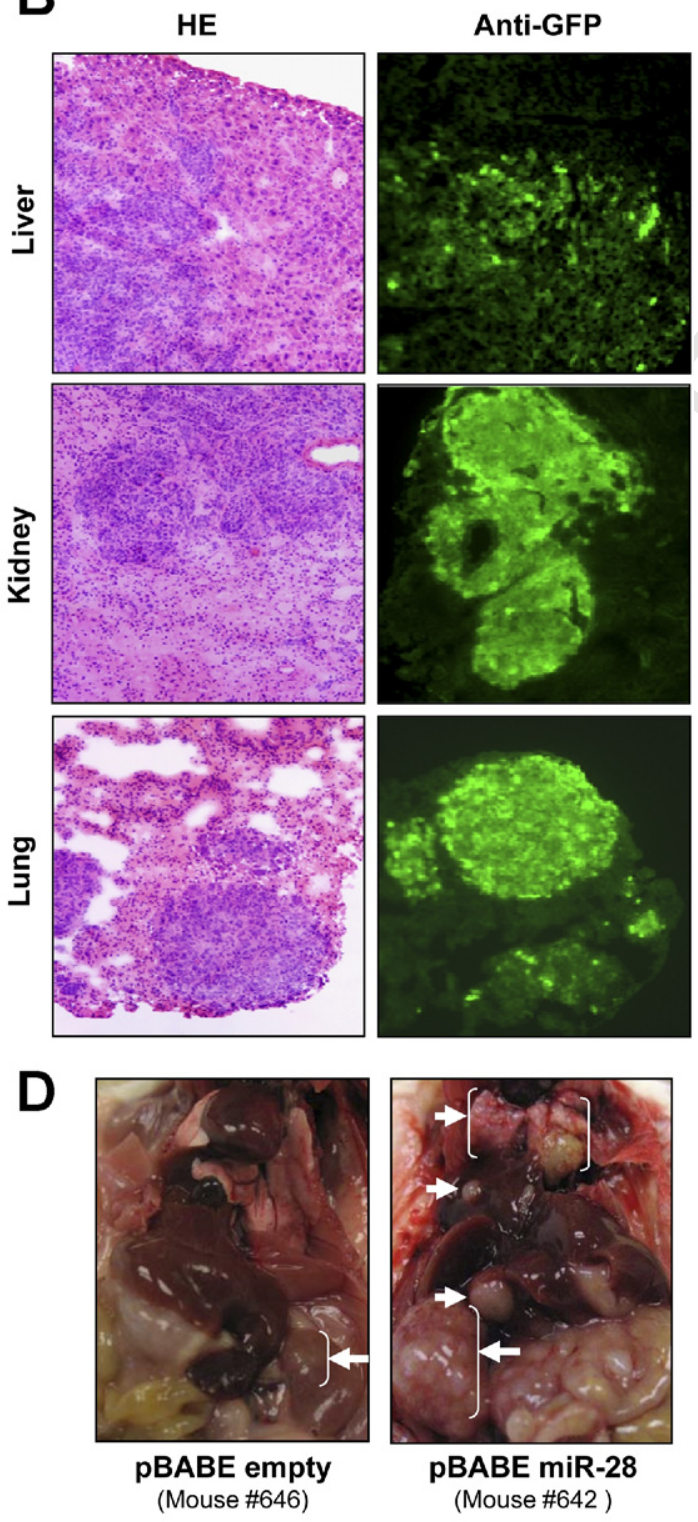

406

407

408

409

410

411

412

413

414

415

416

417

418

419

420

421

422

423

424

425

426

427

428

429

430

431

432

433

434

435

436

437

438

439

440

441 show the sites with metastasis (white arrows) found in $>30 \%$ of each group of mice.
CRC in detail. This study provides evidence that strandspecific $5 \mathrm{p}$ and $3 \mathrm{p}$ miRNAs have distinct functions (Figure $6 F$ ). Concordantly with the role of a tumor-suppressor gene, miR-28-5p suppressed cell proliferation, causing apoptosis and G1 arrest in the cell cycle; however, miR-28-3p had no effect on proliferation in vitro. Therefore, the overall effect in vivo was, as expected, a significant decrease in tumor volume. In contrast, miR-28-5p and miR28-3p caused opposite effects in migration and invasion in vitro. The miR-28-injected mice developed more metastases than did the control mice, which is in agreement with the in vitro effect observed for miR-28-3p-overexpressing cells. To our knowledge, only 2 studies have addressed the distinct roles of $5 \mathrm{p}$ and $3 \mathrm{p}$ strands, but none of them have investigated the in vivo effect or the distinct targeting mechanisms in detail. These studies showed the different effects of miR-125a-3p and miR-
$125 a-5 p$ in lung cancer cells ${ }^{19}$ and miR-34c-3p and miR$34 c-5 p$ in the cervical tumor cell line SiHa. ${ }^{21}$

Recently, Yang et al identified the erythroid 2-related factor 2 as a target of miR-28 in breast cancer. ${ }^{22}$ To understand the underlying mechanisms of miR-28, we searched for miRNA targets (Figure $6 F$ ). Cyclin D1, encoded by the CCND1 gene, is a well-known oncogene that is overexpressed in several types of tumors, including CRC. ${ }^{23}$ This protein is a key player in cell-cycle regulation, in particular in the G1-S phase transition, ${ }^{24,25}$ and its inhibition reduces growth and tumorigenicity in human colon cancer cells. ${ }^{26}$ We found that miR-28-5p, but not miR-28-3p, targets cyclin D1. This is in agreement with the biological functions of miR-28-5p, as only miR-28-5p and not miR-28-3p caused G1 arrest. Although cyclin D1 protein levels were decreased in miR-28$5 \mathrm{p}$-transfected cells, it remains to be determined whether this is a consequence of a direct miR::mRNA interaction or 
Figure 6. miR-28-5p targets cyclin D1 and HoxB3, and miR28-3p targets Nm23-H1. Western blot analysis shows (A) cyclin D1, (B) HoxB3, and (C) Nm23-H1 expression in scrambled, miR-28$5 p$, and miR-28-3p transfected HCT116 cells. Expression levels were normalized for vinculin or glyceraldehyde-3-phosphate dehydrogenase (GAPDH) protein levels and were compared with the scrambled negative control transfection $(=1)$. $(D, E)$ The predicted miRNA::mRNA interaction sites are shown in the top panels. The bottom panels show luciferase activity for the predicted interaction sites (D) PGL3HOXB3-WT constructs cotransfected with scrambled negative control $(n=1)$ or miR-28-5p and (E) PGL3-NM23-H1-WT construct cotransfected with scrambled negative control $(n=1)$ or miR-28-3p. The same experiment was also performed using constructs with a mutated interaction site-PGL3-HOXB3-Mut and PGL3-NM23-H1-Mut. Values represent the mean \pm standard deviation of 2 independent experiments performed in 4 replicates $\left({ }^{*} P<.01\right.$, Student $t$ test). $(F)$ The proposed mechanism for miR28-5p and $-3 p$ function in $C R C$ is shown.

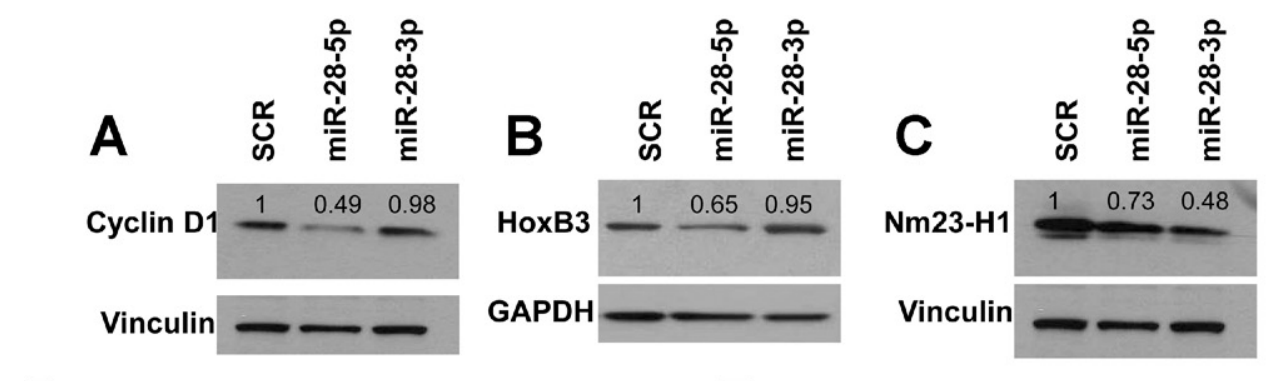

D

hsa-miR-28-5p 3'GAGUUAUCUGACACUCGAGGAA 5' HOXB3 5'AAAAGGCAUGAACUCAGCUCCUA3' hsa-miR-28-3p 3'AGGUCCUCGAGUGUUAGAUCAC5'

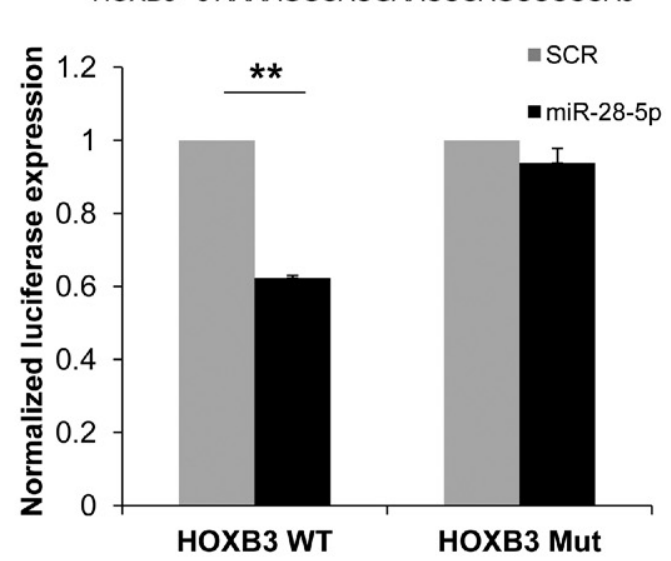
NM23-H1 5'CCAGGCTGTAGGAAATCTAGTT3'

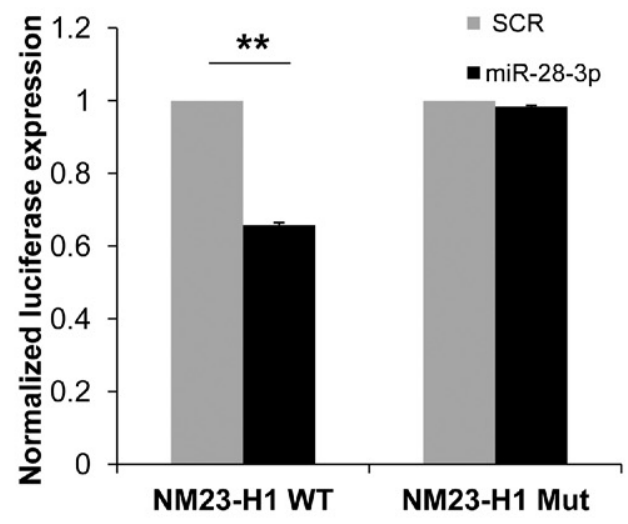

$\mathbf{F}$ an indirect effect through miR-28-5p targeting of other mRNAs in pathways where cyclin D1 is involved. The miR-28-5p::CCND1 binding site predicted in silico showed a slight luciferase reduction that was not abrogated by the binding site mutation, showing that at least in this site there is no direct interaction. However, and although not predicted by our program's analysis, we do not exclude the possibility that other miR-28-5p::CCND1 binding sites might exist. In addition, we also found HOXB3 to be a target of miR-28-5p. HOXB3 has been described as being significantly overexpressed in colon cancer. ${ }^{27}$ Although the role of $\mathrm{HOXB3}$ in colon cancer has not been explored, Palakurthy et al described a mechanism by which $H O X B 3$ exerts it oncogenic role, showing that it is essential for epigenetic silencing of the tumor-suppressor $R A S S F 1 A,{ }^{28}$ the promoter of which is hypermethylated in colon tumors. ${ }^{29}$ These authors also demonstrate in a lung cancer cell line that HOXB3 increases tumor growth both in vitro and in vivo. ${ }^{29}$ In addition, HOXB3 has been demonstrated to regulate cellular proliferation of hematopoietic stem cells ${ }^{30}$ and of Rat- 1 cell line. ${ }^{31}$ The interaction between miR-28-5p and HOXB3 occurs through a direct binding as demonstrated by the luciferase assay results. Our data demonstrate that, in vivo, miR-28 promotes metastasis and that, in vitro, miR-28-3p induces migration and invasion. As miR-28 was reduced in the tumors, we looked for an antimetastatic mRNA as a target, which would suppress metastasis without affecting tumor growth. ${ }^{32}$ Interestingly, we found that miR-28-3p has the capacity of regulating $N M 23-H 1$, the first metastasis-suppressor gene identified. ${ }^{33-35}$ Remarkably, it has been previously 
reported that this gene is overexpressed in colon carcinoma cells, especially in the early stages, and that it limits the invasive potential of human cancer cells without having an effect on proliferation. ${ }^{36}$ In addition, NM23-H1 inhibits liver metastases of colon. ${ }^{37}$

In the future, prospective studies should be performed to address clinical correlations and systematic experiments should be conducted to identify all potential targets that can explain the distinct biological effects.

In conclusion, this is the first study to report downregulation of miR-28 in human tumorigenesis. In CRC, miR-28 suppresses proliferation but activates metastasis; this is a consequence of the distinct roles of the miR-28 hairpin RNA products, miR-28-5p and miR-28-3p. Such information has direct consequences for the design of miRNA gene therapy trials. The manipulation of the expression of specific miRNAs by using the precursor molecules can produce additional clinical effects due to the transcription of $5 p$ and $3 p$ genes with distinct biological effects.

\section{Supplementary Material}

Note: To access the supplementary material accompanying this article, visit the online version of Gastroenterology at www.gastrojournal.org, and at doi:10.1053/ j.gastro.2011.12.047.

\section{References}

1. Jemal A, Bray F, Center MM, et al. Global cancer statistics. CA Cancer J Clin 2011;61:69-90.

2. Jemal A, Siegel R, Xu J, et al. Cancer statistics, 2010. CA Cancer J Clin 2010;60:277-300.

3. Calin GA, Dumitru CD, Shimizu M, et al. Frequent deletions and down-regulation of micro- RNA genes miR15 and miR16 at 13q14 in chronic lymphocytic leukemia. Proc Natl Acad Sci U S A 2002; 99:15524-15529.

4. Almeida MI, Reis RM, Calin GA. MicroRNA history: discovery, recent applications, and next frontiers. Mutat Res 2011;717:1-8.

5. Croce CM. Causes and consequences of microRNA dysregulation in cancer. Nat Rev Genet 2009;10:704-714.

6. Wu WK, Law PT, Lee CW, et al. MicroRNA in colorectal cancer: from benchtop to bedside. Carcinogenesis 2011;32:247-253.

7. Lea MA. Recently identified and potential targets for colon cancer treatment. Future Oncol 2010;6:993-1002.

8. Griffiths-Jones S, Grocock RJ, van Dongen S, et al. miRBase: microRNA sequences, targets and gene nomenclature. Nucleic Acids Res 2006;34(Database issue):D140-D144.

9. Bustin SA, Benes V, Garson JA, et al. The MIQE guidelines: minimum information for publication of quantitative real-time PCR experiments. Clin Chem 2009;55:611-622.

10. Lefever S, Hellemans J, Pattyn F, et al. RDML: structured language and reporting guidelines for real-time quantitative PCR data. Nucleic Acids Res 2009;37:2065-2069.

11. Schmittgen TD, Livak KJ. Analyzing real-time PCR data by the comparative C(T) method. Nat Protoc 2008;3:1101-1108.

12. Pfaffl MW. A new mathematical model for relative quantification in real-time RT-PCR. Nucleic Acids Res 2001;29:e45.

13. Galluzzi L, Aaronson SA, Abrams J, et al. Guidelines for the use and interpretation of assays for monitoring cell death in higher eukaryotes. Cell Death Differ 2009;16:1093-1107.
14. Chaitanya GV, Steven AJ, Babu PP. PARP-1 cleavage fragments: signatures of cell-death proteases in neurodegeneration. Cell Commun Signal 2010;8:31.

15. Girardot M, Pecquet C, Boukour S, et al. miR-28 is a thrombopoietin receptor targeting microRNA detected in a fraction of myeloproliferative neoplasm patient platelets. Blood 2010;116: 437-445.

16. Gottardo F, Liu CG, Ferracin M, et al. Micro-RNA profiling in kidney and bladder cancers. Urol Oncol 2007;25:387-392.

17. Malzkorn B, Wolter M, Liesenberg $\mathrm{F}$, et al. Identification and functional characterization of microRNAs involved in the malignant progression of gliomas. Brain Pathol 2010;20:539-550.

18. Spizzo R, Nicoloso MS, Croce CM, et al. SnapShot: microRNAs in cancer. Cell 2009;137:586-586.e1.

19. Jiang L, Huang Q, Zhang S, et al. Hsa-miR-125a-3p and hsa-miR$125 a-5 p$ are downregulated in non-small cell lung cancer and have inverse effects on invasion and migration of lung cancer cells. BMC Cancer 2010;10:318.

20. Volinia S, Galasso M, Costinean S, et al. Reprogramming of miRNA networks in cancer and leukemia. Genome Res 2010;20: 589-599.

21. López JA, Alvarez-Salas LM. Differential effects of miR-34c-3p and miR-34c-5p on SiHa cells proliferation apoptosis, migration and invasion. Biochem Biophys Res Commun 2011;409:513-519.

22. Yang $M$, Yao $Y$, Eades $G$, et al. MiR-28 regulates Nrf2 expression through a Keap1-independent mechanism. Breast Cancer Res Treat 2011;129:983-991.

23. Arber N, Hibshoosh $\mathrm{H}$, Moss SF, et al. Increased expression of cyclin D1 is an early event in multistage colorectal carcinogenesis. Gastroenterology 1996;110:669-674.

24. Diehl JA. Cycling to cancer with cyclin D1. Cancer Biol Ther 2002; 1:226-231.

25. Fu M, Wang C, Li Z, et al. Mini review: cyclin D1: normal and abnormal functions. Endocrinology 2004;145:5439-5447.

26. Arber N, Doki Y, Han EK, et al. Antisense to cyclin D1 inhibits the growth and tumorigenicity of human colon cancer cells. Cancer Res 1997;57:1569-1574.

27. Kanai M, Hamada J, Takada M, et al. Aberrant expressions of HOX genes in colorectal and hepatocellular carcinomas. Oncol Rep 2010;23:843-851.

28. Palakurthy RK, Wajapeyee N, Santra MK, et al. Epigenetic silencing of the RASSF1A tumor suppressor gene through HOXB3-mediated induction of DNMT3B expression. Mol Cell 2009;36:219230.

29. Lee S, Hwang KS, Lee HJ, et al. Aberrant CpG island hypermethylation of multiple genes in colorectal neoplasia. Lab Invest 2004 84:884-893.

30. Björnsson JM, Larsson N, Brun AC, et al. Reduced proliferative capacity of hematopoietic stem cells deficient in Hoxb3 and Hoxb4. Mol Cell Biol 2003;23:3872-3883.

31. Krosl J, Baban S, Krosl G, et al. Cellular proliferation and transformation induced by HOXB4 and HOXB3 proteins involves cooperation with PBX1. Oncogene 1998;16:3403-3412.

32. Lee JH, Marshall JC, Steeg PS, et al. Altered gene and protein expression by $\mathrm{Nm} 23-\mathrm{H} 1$ in metastasis suppression. Mol Cell Biochem 2009;329:141-148.

33. Steeg PS, Bevilacqua G, Kopper L, et al. Evidence for a novel gene associated with low tumor metastatic potential. J Natl Cancer Inst 1988;80:200-204.

34. Steeg PS, Bevilacqua G, Pozzatti R, et al. Altered expression of NM23, a gene associated with low tumor metastatic potential, during adenovirus 2 Ela inhibition of experimental metastasis. Cancer Res 1988;48:6550-6554.

35. Marshall JC, Collins J, Marino N, et al. The Nm23-H1 metastasis suppressor as a translational target. Eur J Cancer 2010;46: 1278-1282.

36. Boissan M, De Wever O, Lizarraga F, et al. Implication of metastasis suppressor NM23-H1 in maintaining adherens junctions and 
588 AQ: 1 Reprint requests

589 AQ: 2 Address requests for reprints to: George A. Calin, XX, Department limiting the invasive potential of human cancer cells. Cancer Res 2010;70:7710-7722.

37. Suzuki E, Ota T, Tsukuda K, et al. nm23-H1 reduces in vitro cell migration and the liver metastatic potential of colon cancer cells by regulating myosin light chain phosphorylation. Int J Cancer 2004;108:207-211.

Received July 22, 2011. Accepted December 27, 2011. of Experimental Therapeutics, The University of Texas MD Anderson Cancer Center, 1515 Holcombe Boulevard, Houston, Texas 77030, USA. e-mail: gcalin@mdanderson.org; fax: (713) 745-4528.

AQ: 3 Acknowledgments

The authors thank Sue Moreau from the Department of Scientific Publications at The University of Texas MD Anderson Cancer Center for English language editing of the manuscript.

Author contributions: Study concept and design: M.I.A., P.A.Z, G.A.C. Acquisition of data: M.I.A., L.Z., X.Z. Drafting of the manuscript: M.I.A., M.N., R.S., M.F., R.M.R., P.A.Z, G.A.C. Analysis and interpretation of data: M.I.A., M.N., R.S., R.M., P.A.Z, G.A.C. Critical revision of the manuscript for important intellectual content: M.I.A., M.N., R.S., M.F., R.M.R., P.A.Z, G.A.C. Statistical analysis: M.I.A., C.I., L.X. Obtained funding: G.A.C. Administrative, technical, or material support: R.G., I.V., F.F., M.F., G.L. Study supervision: G.A.C.

Drs Nicoloso and Spizzo are currently at the Division of Experimental Oncology, CRO, National Cancer Institute, Aviano, Italy.

\section{Conflicts of interest}

The authors disclose no conflicts.

\section{Funding}

M.I.A. is supported by a PhD fellowship (SFRH/BD/47031/2008) from Fundação para a Ciência e Tecnologia, Portugal. G.A.C. is supported as a fellow by The University of Texas MD Anderson Cancer Center Research Trust, The University of Texas System Regents Research Scholar, and the Chronic Lymphocytic Leukemia Global Research Foundation. Work in Dr Calin's laboratory is supported in part by grants from the National Institutes of Health (CA135444), the US Department of Defense, the Pancreatic Cancer Action Network (2009 Seena Magowitz AACR Pilot Grant), and the US-European Alliance for the Therapy of Chronic Lymphoid Leukemia. STR DNA fingerprinting was done by the Cancer Center Support grant funded Characterized Cell Line core, NCI \# CA16672. 


\section{Supplementary Methods}

\section{Microsatellite Analysis}

Microsatellite analysis was performed on DNA extracted from frozen tissue samples by a standard phenol-chloroform procedure. MSI was evaluated with a fluorescence-based PCR method using the 5 markers of the Bethesda panel (ie, D5S346, D17S250, D2S123, BAT25, and BAT26) plus BAT40. Analysis of PCR products was done with an automated DNA sequencer. Tumors were classified as MSS, MSI-L, and MSI-H according to the guidelines of the International Workshop of Bethesda. ${ }^{1}$

\section{RNA and Protein Extraction}

RNA was isolated using Trizol reagent (Invitrogen), according to manufacturer's instructions. RNA quantity and purity was assessed with NanoDrop ND1000 (Thermo Fisher Scientific, Wilmington, DE). RNA integrity was analyzed by gel electrophoresis. RNA samples were denaturated at $70^{\circ} \mathrm{C}$ for 5 minutes, immediately placed on ice, and loaded on an agarose gel stained with ethidium bromide. Intensity of the $18 \mathrm{~S}$ and $28 \mathrm{~S}$ bands was examined.

Total protein extracts were prepared in ice-cold lysis buffer $(0.5 \%$ Nonidet P- $40,250 \mathrm{mM}$ sodium chloride, 50 mM HEPES, $5 \mathrm{mM}$ EDTA, and $0.5 \mathrm{mM}$ ethylene glycolbis( $\beta$-aminoethyl ether)- $N, N, N^{\prime}, N^{\prime}$-tetraacetic acid) containing phosphatase inhibitor cocktail 2 (Sigma-Aldrich, St Louis, MO), protease inhibitor (Clontech, Mountain View, CA), and dithiothreitol (Invitrogen).

\section{Reverse Transcription Quantitative Real- Time PCR}

miRNA expression was evaluated using TaqMan miRNA assays (Applied Biosystems). Briefly, complementary DNA was synthesized using RNA as a template, genespecific stem-loop Reverse Transcription primer, and the TaqMan microRNA reverse-transcription kit (Applied Biosystems). Quantitative real-time PCR was carried out in a CFX384 real-time system (Bio-Rad, Hercules, CA) using complementary DNA, TaqMan probe, and TaqMan universal PCR master mix (Applied Biosystems). Experiments were performed in duplicate and normalized to small nuclear RNA U6, which was used as an internal control. Relative expression levels were calculated using the comparative cycle threshold method. Stability of the reference gene between samples was analyzed. PCR efficiency was determined using the formula: Efficiency $=10^{-1 / \text { slope }}-1$.

\section{Cell Culture, STR DNA Fingerprinting, and miRNA Mimics Transfection}

Human CRC HCT116, RKO, and SW480 cell lines (purchased from American Type Culture Collection, Manassas, VA) were grown as suggested by the supplier. Cells were cultured at $37^{\circ} \mathrm{C}$ in $5 \% \mathrm{CO}_{2}$.
All cell lines used in this study were validated by STR DNA fingerprinting using the AmpF $\ell$ STR Identifiler kit, according to manufacturer instructions (Applied Biosystems). The STR profiles were compared with known ATCC fingerprints (ATCC.org), to the Cell Line Integrated Molecular Authentication database version 0.1.200808 (http:// bioinformatics.istge.it/clima/), ${ }^{2}$ and to the MD Anderson fingerprint database. STR profiles of HCT116, RKO, and SW480 cell lines matched known DNA fingerprints and were unique.

Pre-miRNA miRNA precursor molecules for hsa-miR28-5p and hsa-miR-28-3p and pre-miR miRNA precursor scrambled negative control (SCR) \#2 were purchased from Ambion (Austin, TX). Transfections were performed using $50 \mathrm{nM}$ miRNA specific-strand precursor molecules or control and Lipofectamine 2000 reagent (Invitrogen), according to manufacturer's instructions. RNA and proteins were collected at 48 hours after transfection. miRNA transfection efficiencies were evaluated by reverse transcription quantitative real-time PCR.

\section{3-(4,5-Dmethylthiazol-2-yl)-2,5- Diphenyltetrazolium Bromide) Assay}

We seeded $5 \times 10^{3}$ HCT116 cells transfected with either SCR or miR-28-5p in a 96-well plate in 8 replicates for each condition. At each time point $(0,24,48,72$, and 96 hours post transfection), the colorimetric reagent was added to the cells. After 2-hour incubation at $37^{\circ} \mathrm{C}$, dimethylsulfoxide was added. Proliferation was assessed by measuring absorbance at $580 \mathrm{~nm}$ using the SpectraMax Plus ${ }^{384}$ microplate reader (Molecular Devices, Sunnyvale, CA). Experiment was performed 2 times independently.

\section{Apoptosis Quantification}

Protein levels of the apoptotic molecular marker PARP1, full-length, and cleavage PARP1 forms were assessed by Western blot analysis using PARP antibody (9542) from Cell Signaling Technology (Danvers, MA) in the HCT116 and RKO cell lines transfected with SCR, miR-28-5p, or miR-28-3p. Relative intensity of bands observed by Western blotting was obtained using ImageJ software (http://imagej.nih.gov/ij/). In addition, caspase $3 / 7,8$, and 9 activity was measured.

\section{Caspase 3/7, 8, and 9 Activity}

Caspase activity was measured using Caspase-Glo 3/7 Assay Systems, Caspase-Glo 8 Assay Systems, and Caspase-Glo 9 Assay Systems (Promega Corporation, Madison, WI) in HCT116 cells transfected with SCR, miR-28-5p, or miR-28-3p. The assay was performed 48 hours post transfection according to manufacturer's instructions, and luminescence was measured in a POLARstar OPTIMA microplate reader (BMG Labtech, Ortenberg, Germany). 


\section{Cell-Cycle Analysis by Flow Cytometry}

For fluorescent-activated cell sorting analysis, 6 $\times 10^{5}$ HCT 116 cells transfected with either SCR, miR28-5p, or miR-28-3p were plated onto 6-well plates. After 48 hours, cells were collected and fixed with 70\% ice-cold ethanol. Cells were stained with a solution containing $0.05 \mathrm{mg} / \mathrm{mL}$ propidium iodide (Sigma-Aldrich) and $0.1 \mathrm{mg} / \mathrm{mL}$ RNase A (Roche, Indianapolis, IN) in phosphate-buffered saline. Cell-cycle analysis was performed in a FACSCalibur flow cytometer (Becton Dickinson, San Jose, CA). Results were analyzed using ModFit LT software.

\section{In Vitro Cell Migration and Invasion Assays}

After 24- or 48-hour incubation (for migration and invasion assay, respectively) at $37^{\circ} \mathrm{C}$ with $5 \% \mathrm{CO}_{2}$, cells were fixed with paraformaldehyde (USB Corporation, Cleveland, $\mathrm{OH}$ ). Cells on the upper surface of the chamber (nonmigratory cells) were removed using cotton swabs, and cells on the bottom surface (migratory cells) were stained with crystal violet in 20\% methanol for 20 minutes. Finally, $30 \%$ acetic acid was added to dissolve the crystal violet and absorbance was measured in a SpectraMax Plus ${ }^{384}$ spectrophotometer (Molecular Devices) at $590 \mathrm{~nm}$.

\section{Establishment of miR-28-Expressing Cell Line:} Cell Transduction With Retroviral Vector

A PCR fragment of $483 \mathrm{nt}$ that included the human miR-28 precursor and flanking sequences was amplified using primers with BamHI and EcoRI endonucleases restriction sites (Supplementary Table 4). pBABE-puro retroviral plasmid and miR-28-containing fragment were digested with BamHI and EcoRI enzymes and ligated using T4 DNA ligase (New England Biolabs, Ipswich, MA). Constructs were checked by direct sequencing. The retroviral plasmid pBABEmiR28 was transiently transfected together with pVSV-G vector into GP2-293 cells using Lipofectamine 2000 reagent (Invitrogen). The retroviral plasmid pBABE-empty was used as a control. Cells were fed with fresh medium the day after transfection. Viral supernatant was collected 3 days after transfection, filtered through $0.45-\mu \mathrm{m}$ pore, and supplemented with Sequa-brene (Sigma-Aldrich). HCT116 cells, which are known to have metastatic potential, ${ }^{3}$ were infected and selected using puromycin. Successful establishment of HCT116-pBABE-miR28 cell line was verified by reverse transcription quantitative real-time PCR.

\section{Cell Transduction With Lentiviral Vector}

As pBABE-puro does not contain green fluorescent protein marker, and to facilitate the detection of the human colon cancer cells in the in vivo studies, HCT116pBABE-empty and HCT116-pBABE-miR28 cells were transduced in parallel with empty pRRL-CMV-PGK-GFP-
WPRE (Tween) lentiviral vector. Briefly, pTween vector was cotransfected with the packaging vector $\mathrm{PCM}$ VDR8.74 and the envelope vector pMD.G into 293FT cells using Lipofectamine 2000 reagent. Forty-eight hours after transfection, supernatant containing the virus was collected, filtered through $0.45-\mu \mathrm{m}$ pore, and supplemented with Sequa-brene. HCT116-pBABE-empty and HCT116-pBABE-miR28 were incubated with the viral soup for 45 minutes and centrifuged at $32^{\circ} \mathrm{C}$ at 1800 $\mathrm{rpm}$, plus another 1 hour and 15 minutes in the incubator at $37^{\circ} \mathrm{C}$. Infection efficiency was evaluated by flow cytometry by detecting the percentage of green fluorescent protein-positive cells ( $>85 \%$ ).

\section{miRNA Target Prediction}

We performed in silico analysis to determine miR28-5p- and miR-28-3p-predicted targets using an in-house Perl script that scans the databases for the algorithms PITA (http://genie.weizmann.ac.il/pubs/mir07), TargetScan (http:// www.targetscan.org), miRanda (http://www.microrna.org), and RNA22 (http://cbcsrv.watson.ibm.com/) for target identification. miR-28 sequence annotation was obtained from the miRBase database (http://www.mirbase.org/) (Supplementary Table 4).

\section{Western Blot Analysis for miRNA Targets}

Proteins were collected 48 hours after cells were transfected with SCR, miR-28-5p, or miR-28-3p. Bradford assay was used to measure protein concentration. Proteins were separated by polyacrylamide gel (Bio-Rad) electrophoresis and were transferred to $0.2-\mu \mathrm{m}$ nitrocellulose membranes (Bio-Rad). The following antibodies were used: anti-cyclin D1 (sc-20044), anti-HoxB3 (sc28606), and anti-Nm23-H1 (sc-343) all from Santa Cruz Biotechnology (Santa Cruz, CA). Proteins were detected by chemiluminescence. Anti-glyceraldehyde-3-phosphate dehydrogenase from Cell Signaling Technology or anti-vinculin (sc-5573) from Santa Cruz Biotechnology were used as normalizers.

\section{Luciferase Reporter Assays}

Fragments of about $200 \mathrm{nt}$ that contained the miR-28-5p and miR-28-3p putative binding sites were amplified by PCR using primers containing the XbaI restriction enzyme site (Supplementary Table 4). PCR products were purified, digested, and directly cloned into the Xbal site of the pGL3 control vector (Promega Corporation, Madison, WI) located downstream of the firefly luciferase reporter gene. The QuikChange II XL sitedirected mutagenesis kit (Agilent Technologies, Santa Clara, CA) was used to generate mutations in the miRNA-binding site (Supplementary Table 4).

HCT116 cells were seeded $\left(1 \times 10^{5}\right.$ cells/well $)$ in 24 well plates. After 24 hours, cells were cotransfected with $50 \mathrm{nM}$ SCR, miR-28-5p, or miR-28-3p and 0.4 $\mu \mathrm{g}$ pGL3putative binding site plasmids or pGL3-mutated putative 
binding site plasmids, together with Renilla luciferase construct, which was used as a normalization reference. Transfections were performed in OPTI-MEM I (Invitrogen) using Lipofectamine 2000 reagent. Cells were lysed 48 hours after transfection, and luciferase activity was measured using a dual-luciferase reporter assay system (Promega Corporation) in the veritas microplate luminometer (Turner Biosystems). Two independent experiments were performed with 4 replicates each. Normalized relative luciferase activity was calculated by the formula: [firefly luciferase]/[Renilla luciferase] activity. All constructs were confirmed by direct sequencing using an ABI 3730xl DNA analyzer sequencer (Applied Biosystems).

\section{Supplementary References}

1. Boland CR, Thibodeau SN, Hamilton SR, et al. A National Cancer Institute Workshop on Microsatellite Instability for Cancer Detection and Familial Predisposition: development of international criteria for the determination of microsatellite instability in colorectal cancer. Cancer Res 1998;58:5248-5257.

2. Romano P, Manniello A, Aresu 0 , et al. Cell line data base: structure and recent improvements towards molecular authentication of human cell lines. Nucleic Acids Res 2009;37:D925D932.

3. Rajput A, Dominguez San Martin I, Rose R, et al. Characterization of HCT116 human colon cancer cells in an orthotopic model. J Surg Res 2008;147:276-281. 


\section{IIR-28-5P}

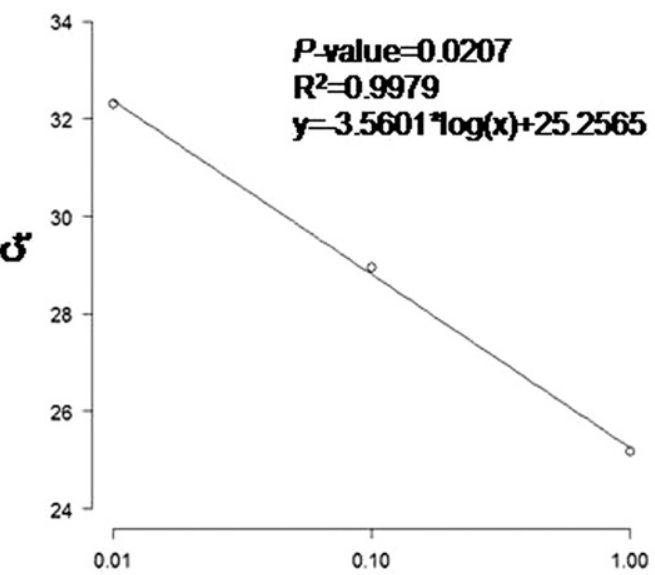

Log cDwAdtions

SRNAU6

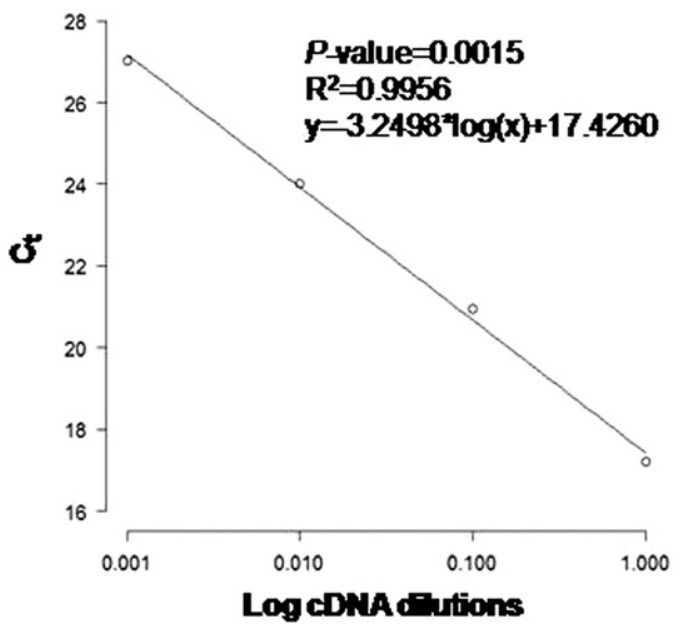

IIR-28-3p

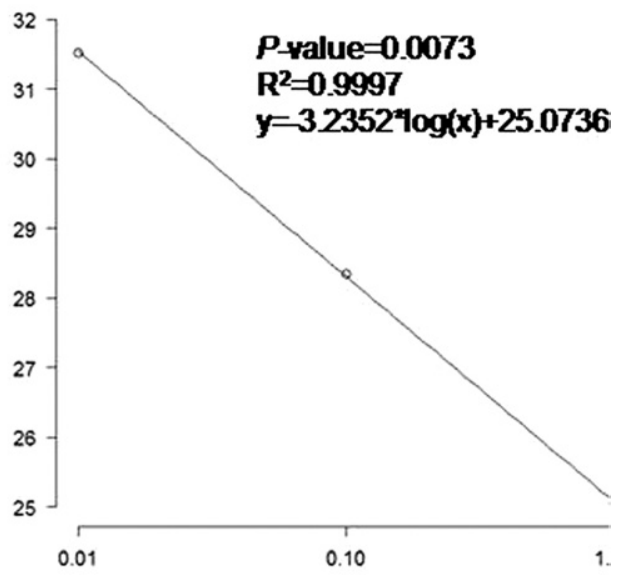

Log CDNA ditions
806

807

808

809

810

811

812

813

814

815

816

817

818

819

820

821

822

823

824

825

826

827

828

829

830

831

832

833

834

835

836

837

838

839

840

841

842

843

844

845

846

847

848

849

850

851

852

853

854

855

856

857

858

859

860

861

nuclear RNA U6 (snRNA U6) variations between samples from norma colon and tumor tissue. There are no differences in small nuclear RNA U6 expression between the 2 groups ( $P=.41$, Mann-Whitney-Wilcoxon test). 

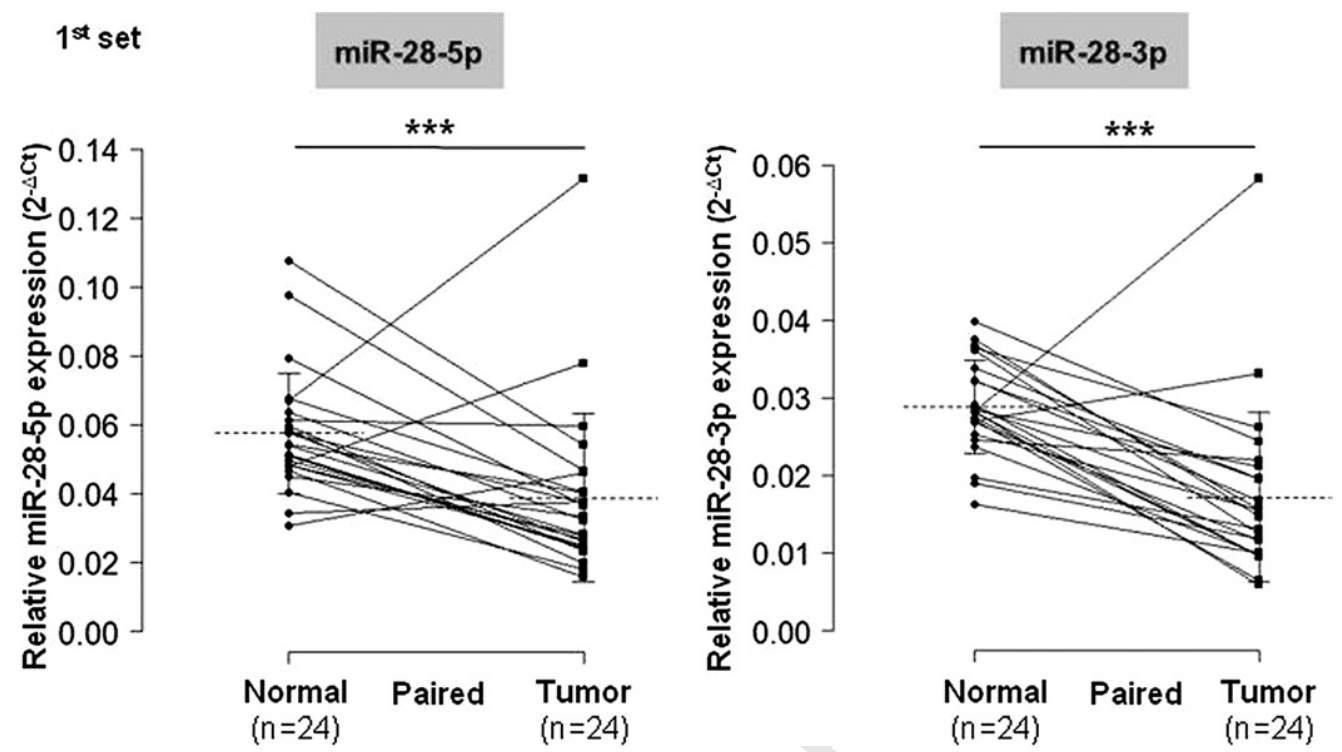

Supplementary Figure 3. Twenty-four normal specimens from the first set of patients were paired with colon cancer tissues from the same patient. All values of miRNA expression levels were normalized by small nuclear RNA U6. Significant differences were ${ }^{\star \star \star} P<.005$ using paired $t$ test.
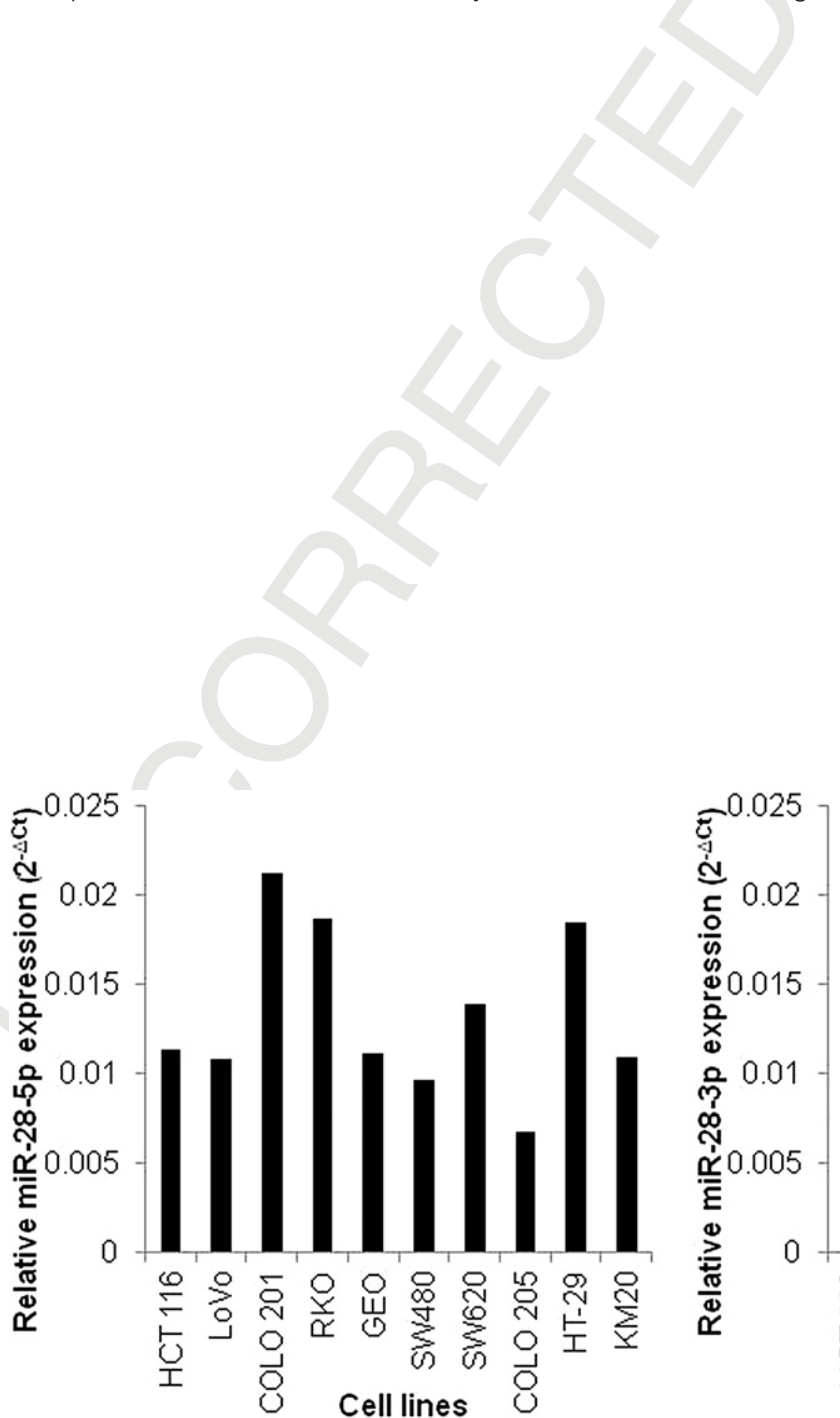

Supplementary Figure 4. Endogenous levels of miR-28-5p and miR-28-3p in 10 colon cancer cell lines. Small nuclear RNA U6 was used as a normalizer. 


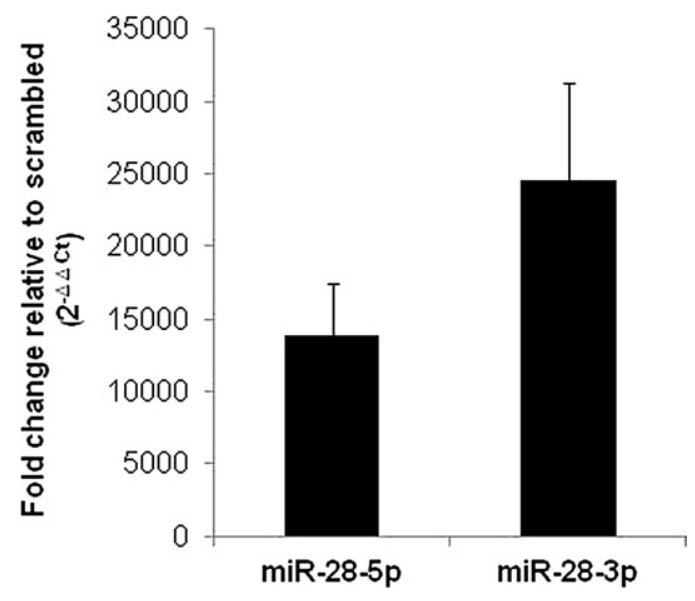

Supplementary Figure 5. miR-28-5p and miR-28-3p levels were measured by quantitative real-time PCR after transient transfection of HCT116 cells with miR-28-5p and miR-28-3p precursors. Values were normalized to small nuclear RNA U6 and are representative of 2 independent experiments. Values shown are relative to negative control.

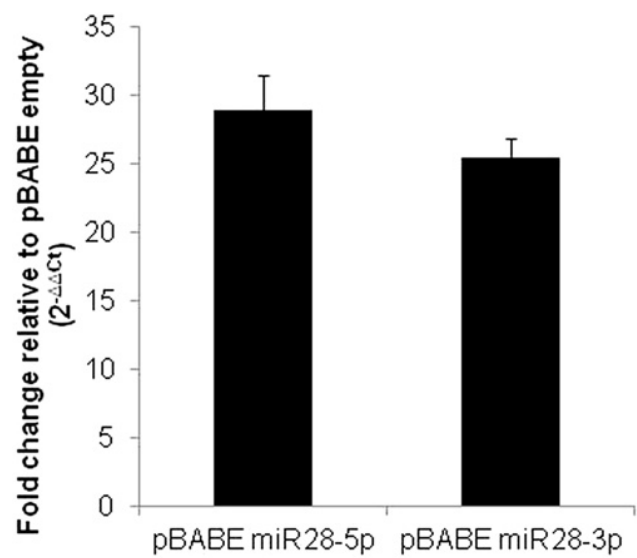

Supplementary Figure 7. miR-28-5p and miR-28-3p levels were measured by quantitative real-time PCR after generating the stable clone pBabe-miR-28 in the HCT116 cell line. Values were normalized to small nuclear RNA U6 and are representative of 2 independent experiments. Values shown are relative to the control pBABE-empty $(n=1)$.
918

919

920

921

922

923

924

925

926

927

928

929

930

931

932

933

934

935

936

937

938

939

940

941

942

943

944

945

946

947

948

949

950

951

952

953

954

955

956

957

958

959

960

961

962

963

964

965

966

967

968

969

970

Supplementary Figure 6. 3-(4,5-dmethylthiazol-2-yl)-2,5-diphenyltetrazolium bromide proliferation assay in $(A) \mathrm{HCT} 116$ and (B) RKO cell lines. miR-28-5p, but not miR-28-3p, inhibited cell growth compared with SCR. Values represent the mean \pm standard deviation of 8 replicates. (C) Caspase activity was measured in the HCT116 cell line 48 hours after transfection with SCR $(n=1)$, miR-28-5p, or miR-28-3p. 


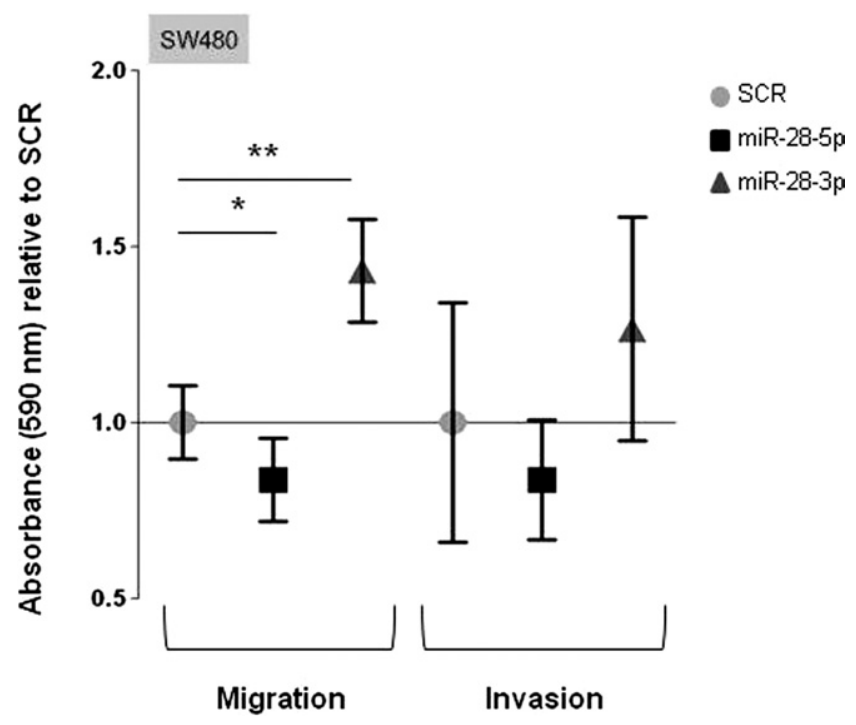

Supplementary Figure 8. Effect of mir-28-5p and miR-28-3p in migration and invasion in vitro in SW480 cell line. Absorbance was measured for cells on the bottom of noncoated and Matrigel-coated Transwell chambers at 24 hours (for migration) and 48 hours (for invasion) after SW480 cells expressing miR-28-5p or miR-28-3p were plated. Results are shown relative to SCR. A representative experiment is shown. Mean of triplicates \pm standard deviation is shown $\left({ }^{\star} P<.05\right.$; ${ }^{* \star} P<.01$, Student $t$ test).
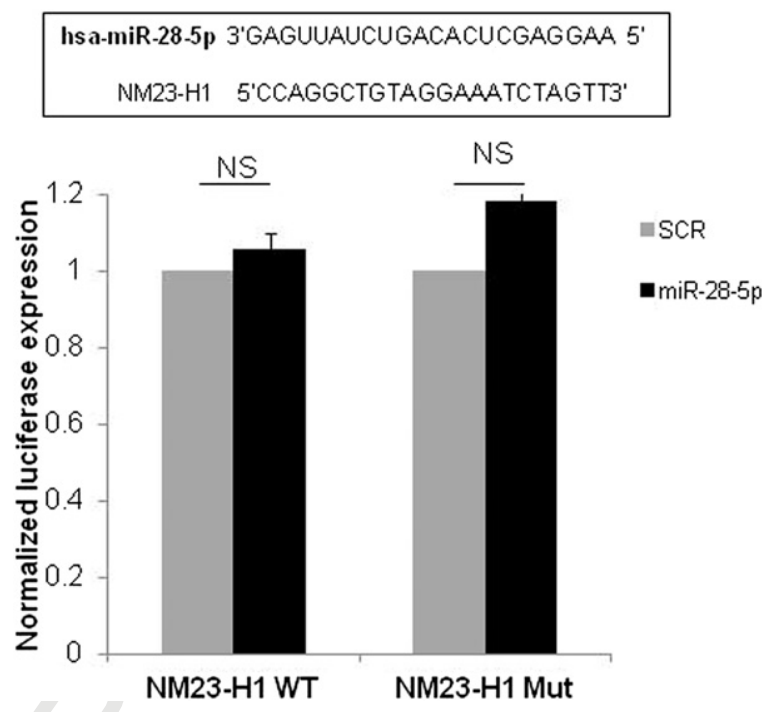

Supplementary Figure 10. Luciferase activity of HCT116 cells cotransfected with scrambled negative control $(n=1)$ or miR-28-5p and PGL3-NM23-H1-WT. Experiment was also performed with a construct in which the binding site was mutated. NS, not statistically significant (Student $t$ test). CCND1 5'CTACTTGGTTTGTGTTCTTCTT3'

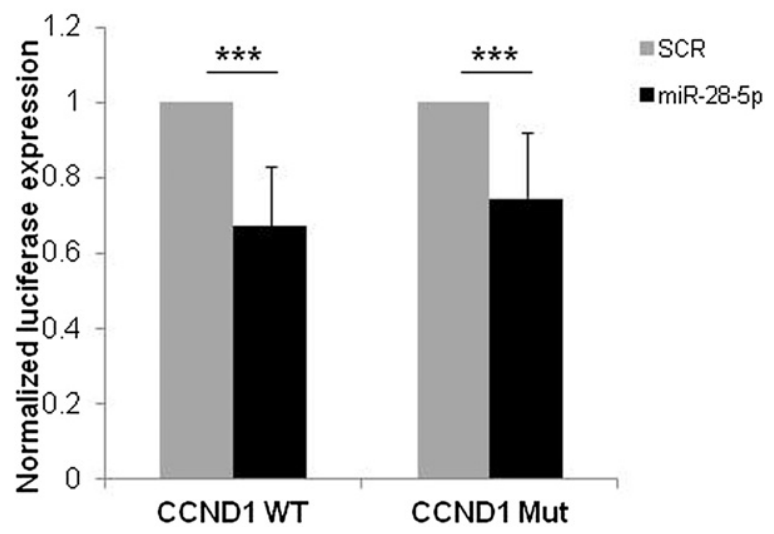

Supplementary Figure 9. Luciferase activity of HCT116 cells cotransfected with scrambled negative control $(n=1)$ or miR-28-5p and PGL3-CCND1-WT. Experiment was also performed with a construct in which the binding site was mutated ${ }^{* * *} P<.005$, Student $t$ test).
1010

1011

1012

1013

1014

1015

1016

1017

1018

1019

1020

1021

1022 

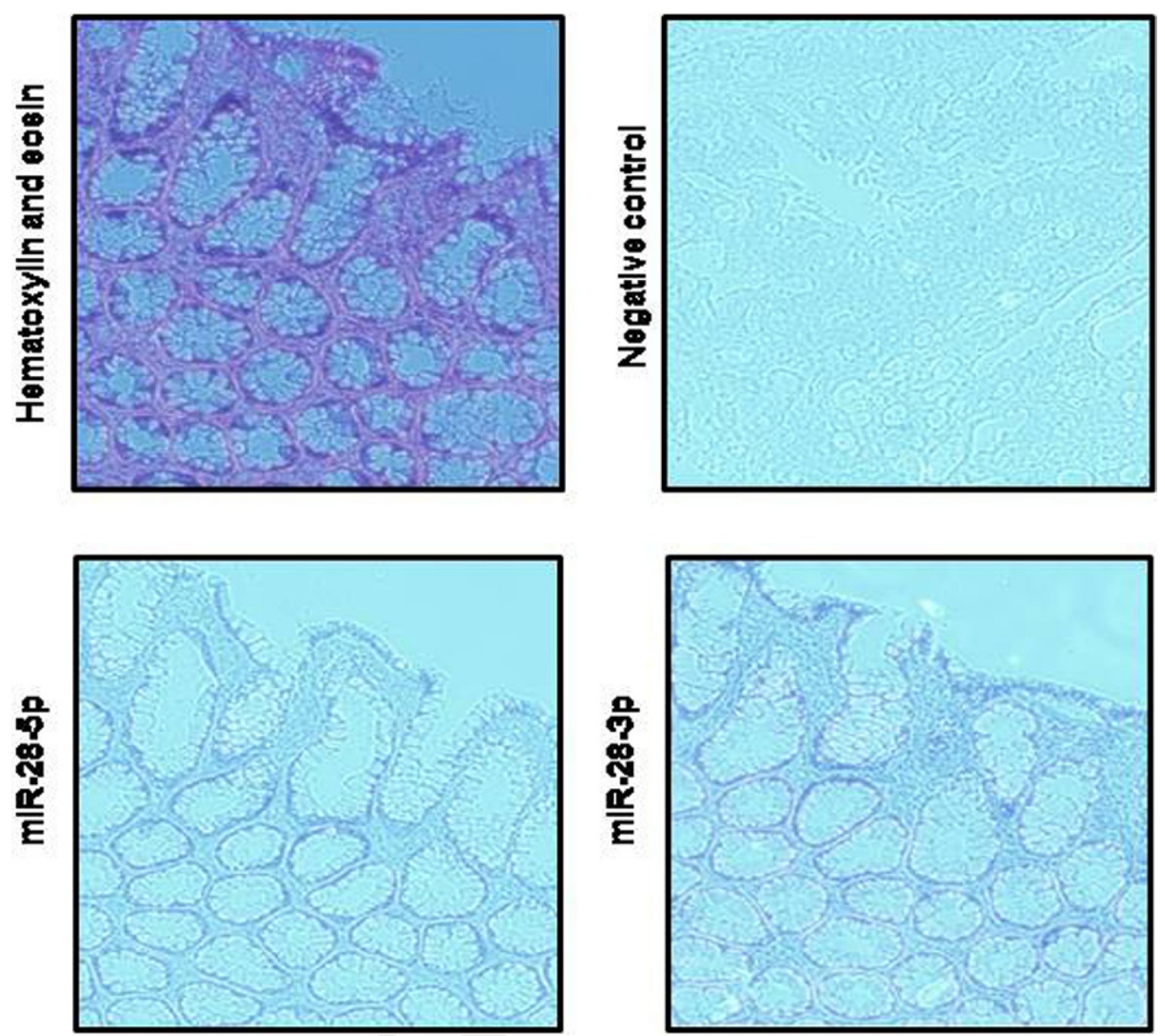

1045

1046

1047

1048

1049

1050

1051

1052

1053

1054

1055

1056

1057

Supplementary Figure 11. In situ hybridization analysis for miR-28-5p and miR-28-3p in normal colon tissue. Frozen tissue sections were digested with proteinase $\mathrm{K}$ and loaded onto Ventan Discovery Ultra. The tissue slides were incubated with double-DIG labeled miRCURY LNA Detection probe and the digoxigenin was detected with a polyclonal anti-DIG antibody and UltraMap Blue anti-Ms Detection Kit. H\&E staining was performed. Microscopy images were obtained with a magnification of $100 \times$.

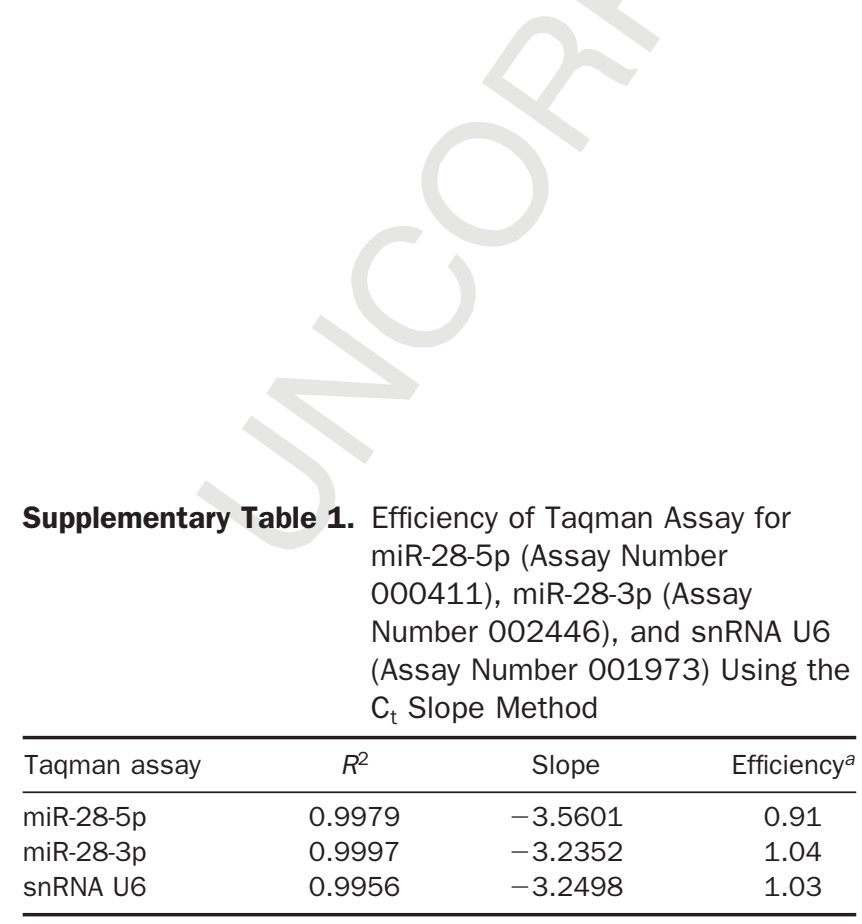

aPCR efficiency was determined using the formula: Efficiency $=$ $10^{-1 / \text { slope }-1 .}$
Supplementary Table 2. miRNA-28-5p and miR-25-3p Expression (Using $\Delta \mathrm{C}_{\mathrm{t}}$ Method) in Normal Colon and Colorectal Cancer Samples for 2 Independent Sets

\begin{tabular}{|c|c|c|}
\hline & Mean & SEM \\
\hline \multicolumn{3}{|l|}{ miR-28-5p } \\
\hline \multicolumn{3}{|c|}{ First set of samples } \\
\hline Normal & 0.058 & 0.003 \\
\hline Tumor & 0.044 & 0.003 \\
\hline MSS & 0.043 & 0.004 \\
\hline $\mathrm{MSI}$ & 0.046 & 0.004 \\
\hline \multicolumn{3}{|c|}{ Second set of samples } \\
\hline Normal & 0.238 & 0.025 \\
\hline Tumor & 0.151 & 0.019 \\
\hline \multicolumn{3}{|l|}{ miR-28-3p } \\
\hline \multicolumn{3}{|c|}{ First set of samples } \\
\hline Normal & 0.029 & 0.001 \\
\hline Tumor & 0.022 & 0.002 \\
\hline MSS & 0.022 & 0.002 \\
\hline $\mathrm{MSI}$ & 0.022 & 0.002 \\
\hline \multicolumn{3}{|c|}{ Second set of samples } \\
\hline Normal & 0.319 & 0.028 \\
\hline Tumor & 0.161 & 0.017 \\
\hline
\end{tabular}

NOTE. Values were normalized to small nuclear RNA U6. SEM, standard error of mean.
1059

1060

1061

1062

1063

1064

1065

1066

1067

1068

1069

1070

1071

1072

1073

1074

1075

1076

1077

1078

1079

1080

1081

1082

1083

1084

1085 
Supplementary Table 3. miR-28-5p Expression in Colorectal Cancer Compared With Normal Colon in 2 Independent Sets of Samples $^{a}$

\begin{tabular}{|c|c|c|c|c|c|c|c|c|}
\hline Gene & Type & $\begin{array}{l}\text { Reaction } \\
\text { efficiency }\end{array}$ & Samples & Expression & Standard error & $95 \% \mathrm{Cl}$ & $\begin{array}{l}P \text { value } \\
(\mathrm{H} 1)\end{array}$ & Result \\
\hline \multirow[t]{3}{*}{ miR-28-5p } & Target & 0.9094 & First set (paired) & 0.620 & $0.389-0.971$ & $0.258-2.380$ & .000 & Down \\
\hline & & & $\begin{array}{l}\text { Second set } \\
\quad \text { (paired) }\end{array}$ & 0.641 & $0.320-1.254$ & $0.173-2.544$ & .003 & Down \\
\hline & & & First set (all) & 0.711 & $0.441-1.209$ & $0.211-2.282$ & .001 & Down \\
\hline U8 & Reference & 1.0309 & & 1 & & & & \\
\hline
\end{tabular}

NOTE. Small nuclear RNA U6 was used as a reference gene.

$\mathrm{Cl}$, confidence interval.

aUsing Pfaffl Method, REST 2009 Software (Qiagen, V2.0.13, http://www.qiagen.com/Products/REST2009Software.aspx?r=8042\#Tabs=t1).

Supplementary Table 4. Sequences of Mature Human miR-28-5p and miR-28-3p According to miRBase, Primers Used to Amplify miR-28, and Primers Used to Generate PGL3 Constructs for Luciferase Assays and to Generate Deletions in the miRNA-Binding Site

\begin{tabular}{ll}
\hline & \\
\hline Mature miRNA & Sequences \\
hsa-miR-28-5p & \\
hsa-miR-28-3p & AAGGAGCUCACAGUCUAUUGAG \\
Primers & CACUAGAUUGUGAGCUCCUGGA \\
mir-28-Fw-BamHI & \\
miR-28-Rv-EcoRI & CGGATCCAGGCCCTTCAAGGACTTTCT \\
Primer for PGL3 construct & CGAATTCACAGAGCTCCTGCTGTGTCA \\
CCND1_Xbal_Fw & \\
CCND1_Xbal_Rv & CGTCTAGAGTCCCACTCCTACGATACGC \\
HOXB3_Xbal_Fw & CGTCTAGACTTGCCTCAAAGTCCTGCTT \\
HOXB3_Xbal_Rv & CGTCTAGAAAGGACATTGTGTTTCCTGTCA \\
NM23_Xbal_Fw & CGTCTAGACAAAGAAAGTTCCAAGAGGGAAT \\
NM23_Xbal_Rv & CGTCTAGAGCAGACCACATTGCTTTTCA \\
Primers for mutagenesis & CGTCTAGAAACCAACTCAATGAATCCTATGC \\
CCND1_Mutagenesis_Fw & \\
CCND1_Mutagenesis_Rv & \\
HOXB3_Mutagenesis_Fw & GGTTCAACCCACAGCTACTTGCATATTCTAAAACCATTCCAT \\
HOXB3_Mutagenesis_Rv & ATGGAATGGTTTAGAATATGCAAGTAGCTGTGGGTTGAACC \\
NM23_Mutagenesis_Fw & GTTCTAAAAGGCATGAACTCATCGTCACTGTATAGTCCTG \\
NM23_Mutagenesis_Rv & CAGGACTATACAGTGACGATGAGTTCATGCCTTTTAGAAC \\
\hline
\end{tabular}

NOTE. Restriction sites for endonucleases are underlined.

1086

1087

1088

1089

1090

1091

1092

1093 